\title{
Philosophiques
}

\section{Le constructivisme piagétien et la théorie de l'équilibration illustrés par la construction de la notion de proportion}

\section{Gérald Noelting}

Volume 4, numéro 2, octobre 1977

Philosophie et psychologie

URI : https://id.erudit.org/iderudit/203071ar

DOI : https://doi.org/10.7202/203071ar

Aller au sommaire du numéro

Éditeur(s)

Société de philosophie du Québec

ISSN

0316-2923 (imprimé)

1492-1391 (numérique)

Découvrir la revue

Citer cet article

Noelting, G. (1977). Le constructivisme piagétien et la théorie de l'équilibration illustrés par la construction de la notion de proportion. Philosophiques, 4(2), 145-194. https://doi.org/10.7202/203071ar d'utilisation que vous pouvez consulter en ligne. 


\section{LE CONSTRUCTIVISME PIAGETIEN ET LA THEORIE DE L'ÉQUILIBRATION ILLUSTRÉS PAR LA CONSTRUCTION DE LA NOTION DE PROPORTION}

par Gérald Noelting

TABLE DES MATIÈRES

Pages

INTRODUCTION $\ldots \ldots \ldots \ldots \ldots \ldots \ldots \ldots \ldots \ldots \ldots \ldots \ldots \ldots$

A. LA THÉORIE CONSTRUCTIVISTE DE PIAGET 147

a) L'interaction entre schème et objet et l'équilibration simple ........................... 147

b) L'origine des "perturbations " et l'équilibration majorante ......................... 150

c) Les trois phases de l'équilibration majorante: $\alpha, \beta, \gamma$

B. ILLUSTRATION DU PROCESSUS D'EQUILIBRA. TION DANS LA CONSTRUCTION DE LA NOTION DE PROPORTION CHEZ L'ENFANT ET L'ADOLESCENT $\ldots \ldots \ldots \ldots \ldots \ldots \ldots \ldots \ldots, 152$

1. L'ÉPREUVE DES CONCENTRATIONS (Forme A) . 152

a) Instrument $\ldots \ldots \ldots \ldots \ldots \ldots \ldots \ldots \ldots \ldots \ldots \ldots \ldots \ldots$

b) Échantillon $\ldots \ldots \ldots \ldots \ldots \ldots \ldots \ldots \ldots \ldots \ldots \ldots$

c) Résultats $\ldots \ldots \ldots \ldots \ldots \ldots \ldots \ldots \ldots \ldots \ldots \ldots \ldots$

2. STRATÉGIE DE RÉSOLUTION DE PROBLÈME ET SYMBOLISME UTILISÉ .................... 157

a) Les composantes d'un item ............ 157

b) Symboles algébriques utilisés pour traduire un item .............................. 159

c) Présentation standardisée d'un item ........ 159

d) Les deux modes $\mathrm{E}$ et I de résolution d'un problème de rapport .................. 159

e) Formalisation des stratégies de résolution caractéristiques de chaque stade ............. 160

3. ANALYSE DES STRATÉGIES AUX DEUX PÉRIODES SUCCESSIVES DE L'ÉLABORATION DU RAISON. NEMENT PROPORTIONNEL $\ldots \ldots \ldots \ldots \ldots \ldots, 160$ 
a) Les deux périodes d'équilibration $\ldots \ldots \ldots \ldots 160$

b) Période d'équilibration I : préopératoire (4.5 à $8-9$ ans) . ......................... 163

c) Période d'équilibration II : opératoire concrète et formelle (8-9 à 15.16 ans) $\ldots \ldots \ldots \ldots \ldots 170$

C. INTERPRÉTATION ET DISCUSSION DES PRO. CESSUS EN JEU DANS LA CONSTRUCTION D'UNE NOTION

1. LA DUALITÉ FONCTIONNELLE ADAPTATIONORGANISATION ..................... 179

a) L'adaptation $\ldots \ldots \ldots \ldots \ldots \ldots \ldots \ldots \ldots . \ldots 179$

b) L'organisation $\ldots \ldots \ldots \ldots \ldots \ldots \ldots \ldots \ldots \ldots \ldots$

2. RELATION ENTRE LE FONCTIONNEMENT DU "SCHÈME " EN ÉQUILIBRE ET LES PROPRIÉTÉS DU " CONCEPT . ......................... 181

3. DÉSÉquilibre APPORTÉ PAR LE MILIEU LORSQUE L'OBJET DEVIENT “ COMPOSÉ " .

4. L'ÉQUILIBRATION MAJORANTE ET LA RÉORGANISATION DU SCHÈME EN FONCTION DU MILIEU 186

a) L'équilibration majorante et la construction d'une hiérarchie ..................... 186

b) Les étapes de l'équilibration majorante ..... 187

c) Illustration par l'expérience décrite ......... 188

d) L'aspect constructiviste de l'équilibration majorante ........................... 190

5. LES DEUX MODALITÉS D'ÉQUILIBRATION : A L'INTÉRIEUR D'UN STADE ET DANS LE PASSAGE D'UN STADE AU SUIVANT .

6. LES QUATRE PHASES DE L'ÉQUILIBRATION MAJORANTE A LA PREMIÈRE PERIODE . . . . . . . 191

7. LES DEUX PÉRIODES DE L'ÉQUILIBRATION MAJORANTE ....................... 192

8. CONCLusion $\ldots \ldots \ldots \ldots \ldots \ldots \ldots \ldots \ldots \ldots . \ldots \ldots$

RÉFÉRENCES ............................ 194 


\section{INTRODUCTION}

“. . . le structuralisme a toujours été solidaire d'un constructivisme auquel on ne saurait refuser le caractère dialectique avec ses signes distinctifs de développements historiques, d'opposition des contraires et de "dépassements ", sans parler de l'idée de totalité commune aux tendances dialectiques autant que structuralistes " (Piaget, 1968, pp. 101-2).

Le " constructivisme " piagétien s'est progressivement dégagé d'une longue pratique de son auteur avec l'expérience, pratique qui alimentait une curiosité constamment en éveil. C'est pourquoi la formulation ultime de sa théorie (Piaget, 1975) est synthétique.

Le livre sur L'Équilibration des structures cognitives (Piaget, 1975 ) est en effet un ouvrage englobant. Il est l'aboutissement d'une longue réflexion. Mais il doit se comprendre aussi à partir des faits observés. Et c'est effectivement parce que l'observation piagétienne est toujours exacte, et reproductible, que ses concepts ont été la source d'investigations nouvelles et fécondes. Cet ouvrage présente l'organisme placé face au milieu, dans une interaction où chacun apporte sa contribution, aboutissant à la construction de totalités nouvelles.

Nous discuterons ici certains aspects du constructivisme piagétien, nous mettrons l'accent sur un certain nombre de points qui le caractérisent, et illustrerons, au moyen d'un exemple concret, les mécanismes que Piaget a décrits.

\section{A. LA THÉORIE CONSTRUCTIVISTE DE PIAGET}

a) L'interaction entre schème et objet et l'équilibration simple.

Dans Biologie et Connaissance, Piaget (1967) oppose deux points de vue quant à l'interprétation de la connaissance : 
"Aristote, dont l'épistémologie s'efforçait de rejoindre celle du sens commun, voyait en la connaissance une prise de possession des "formes" du réel données en dehors de nous ... dans l'acte de connaître le sujet " devient " l'objet ...

"Depuis Kant, au contraire, la connaissance a pu être considérée comme une incorporation ou intégration de l'objet à des formes intérieures au sujet (ou "formes" a priori) de telle sorte que... l'objet " devient " le sujet ou s'identifie à un secteur de son activité connaissante " (p. 70).

Piaget voit la solution de cette opposition dans un tertium : l'« interactionnisme". C'est ce point de vue que nous voulons analyser ici.

L'unité de la conduite, pour Piaget, est déjà structurée, c'est un "schème ". Le schème est une action structurée, accomplie par un organisme, sur un objet qu'il "transforme". Piaget appelle assimilation cette "transformation" de l'objet par un schème de l'organisme.

Mais déjà au niveau de l'activité fonctionnant en équilibre, il y a nécessité d'adapter le schème à l'objet. En effet, l'objet est situé dans l'espace, peut être plus ou moins grand, ses composantes peuvent être plus ou moins éloignées entre elles. La localisation des composantes à l'intérieur du tout, que constitue l'objet, et de cet objet à l'intérieur de l'espace, peut varier d'un objet à l'autre.

Piaget appelle accommodation cette modification du schème en fonction de la localisation des composantes de l'objet, et de l'objet dans l'espace. Cette accommodation n'affectera pas la "structure " du schème en compréhension, mais son extension et sa quantification.

Ainsi, lorsqu'un enfant veut saisir un objet au moyen du schème de préhension, il devra étendre plus ou moins le bras, écarter plus ou moins les doigts, afin d'appréhender l'objet devant lui.

De même un concept générique " chien " se spécifiera pour appréhender " un épagneul ", " un chien berger ", sans perdre son caractère générique "chien ". Chez l'" espèce ", on retrouvera les composantes définies par le "genre", mais localisées ou quantifiées différemment. La spécification ne portera que sur des déterminations de lieu et de quantité, et non de structure. C'est ainsi que 
le schème est bipolaire et que son fonctionnement consiste dans l'interaction entre deux pôles :

1. le pôle d'assimilation, ou générique, ou du sujet ;

2. le pôle d'accommodation, ou spécifique, ou de l'objet.

Dans un autre langage on pourrait dire que la conduite a un niveau central et un niveau périphérique.

C'est ainsi que le schème est une transformation portant sur des objets et comporte ainsi deux composantes :

1. une composante opérative, qui consiste en modifications imposées par l'organisme au milieu ;

2. une composante objectale, qui correspond aux renseignements qui proviennent à l'organisme à partir du substrat lui-même, qui possède des caractéristiques en propre et réagit aux modifications qui lui sont imposées.

Nous appellerons équilibration " simple " cette adaptation du schème à des circonstances locales et diverses de l'objet, qui n'altèrent pas la structure du schème.

Voyons un peu plus dans le détail en quoi consiste cette équilibration "simple ", dont Piaget (1975) distingue deux aspects :

" $1^{\circ}$ En fonction de l'interaction fondamentale de départ entre le sujet et les objets, il y a d'abord l'équilibration entre l'assimilation de ceux-ci à des schèmes d'action et l'accommodation de ces derniers aux objets...

" $2^{\circ} \mathrm{Il}$ y a en second lieu une équilibration à assurer aux interactions entre les sous-systèmes" (p. 14).

Nous retrouvons ici, sous une forme plus élaborée, la distinction que faisait déjà Piaget (1936) entre les deux fonctions invariantes de l'intelligence :

"Il existe, en effet, dans le développement mental, des éléments variables et d'autres invariants...

"Il convient donc d'éviter à la fois le préformisme de la psychologie intellectualiste et l'hypothèse des hétérogénéités mentales. La solution de cette difficulté est précisément à trouver dans la distinction entre les structures variables et les fonctions invariantes.

"Or ces fonctionnements invariants rentrent dans le cadre des deux fonctions biologiques les plus générales : l'organisation et l'adaptation... il y a adaptation lorsque l'organisme se transforme en 
fonction du milieu, et que cette variation a pour effet un accroissement des échanges entre le milieu et lui favorables à sa conservation ... nous pouvons donc dire que l'adaptation est un équilibre entre l'assimilation et l'accommodation.

"L'intelligence est, en effet, assimilation dans la mesure où elle incorpore à ses cadres tout le donné de l'expérience.

"Que la vie mentale soit aussi accommodation au milieu ambiant, cela ne peut faire davantage de doute. L'assimilation ne peut' jamais être pure, parce qu'en incorporant les éléments nouveaux dans les schèmes antérieurs, l'intelligence modifie sans cesse ces derniers pour les ajuster aux nouvelles données.

"Ceci nous conduit à la fonction d'organisation. Du point de vue biologique, l'organisation est inséparable de l'adaptation : ce sont deux processus complémentaires d'un mécanisme unique, le premier étant l'aspect interne du cycle dont l'adaptation constitue l'aspect extérieur ... Pour ce qui est des rapports entre les parties et le tout, qui définissent l'organisation, on sait assez que chaque opération intellectuelle est toujours relative à toutes les autres et que ses propres éléments sont eux-mêmes régis par la même loi. Chaque schème est ainsi coordonné à tous, et constitue lui-même une totalité à parties différenciées . . . L' " accord de la pensée avec les choses" et l'" accord de la pensée avec elle-même " expriment ce double invariant fonctionnel de l'adaptation et de l'organisation" (pp. 11-14).

b) L'origine des "perturbations " et l'équilibration majorante

Cependant l'objet est une totalité autonome de l'organisme, et certains de ses aspects " échappent " au contact direct avec cet organisme. (C'est ainsi que nous interprétons l'origine du déséquilibre.)

Lorsque l'objet devient à son tour actif, ou subit des modifications dans le milieu, par exemple une "composition ", ou une " déformation ", des aspects, auparavant négligés par l'organisme, seront révélés dans cet objet, aspects qui provoqueront des " perturbations " dans l'application du schème par l'organisme. Ces perturbations font sentir leurs effets au niveau structural du schème, et non pas seulement au niveau local ou quantitatif. Ce sont les vicissitudes de l'adaptation de l'organisme à ces perturbations dans l'application de son schème, qui débordent la structure de ce schème, que Piaget appelle "équilibration majorante ". tion :

Piaget (1975) décrit ainsi cette troisième forme d'équilibra. 
" $3^{\circ}$ Il faut en outre considérer à part l'équilibre progressif de la différenciation et de l'intégration, donc des relations unissant des sous-systèmes à une totalité qui les englobe. Cette troisième forme d'équilibration ne se confond pas avec la seconde, puisqu'elle ajoute une hiérarchie aux simples rapports entre collatéraux ... . En ce cas l'intégration en un tout est affaire d'assimilation et la différenciation exige des accommodations; il y a néanmoins conservation mutuelle du tout et des parties et en ce sens assimilations et accommodations réciproques, mais selon une dimension de hiérarchie et non plus seulement collatérale" (p. 15).

\section{c) Les trois phases de l'équilibration majorante : $\alpha, \beta, \gamma$}

L'intérêt de la formulation nouvelle de Piaget (1975) est de dégager, une fois les diverses formes d'équilibration définies, les phases successives de l'équilibration majorante, qu'il distingue en $\alpha, \beta$ et $\gamma$.

"Cela dit, trois conduites principales sont à distinguer...

" $\alpha$ ) Lorsque surgit un fait nouveau, il peut, selon le cas, ne produire aucune modification dans le système ... ou au contraire créer une perturbation... En ces dernières situations la rééquilibration qui se produit à la suite du déséquilibre ainsi provoqué sera obtenue par une conduite dite de type $\alpha$ dans les deux cas suivants. $S$ 'il s'agit d'une petite perturbation voisine du point d'équilibre, la compensation sera obtenue par une simple modification introduite par le sujet en sens inverse de la perturbation en question... Par contre, la seconde réaction de type $\alpha$ interviendra si la perturbation est plus forte ou jugée implicitement telle par le sujet : en ce cas, il l'annulera en la négligeant sans plus ou en l'écartant simplement.

" $\beta$ ) La seconde conduite consistera, au contraire, à intégrer dans le système l'élément perturbateur surgi de l'extérieur, la compensation consistant alors, non plus à annuler la perturbation ou à rejeter l'élément nouveau pour qu'il n'intervienne pas à l'intérieur de l'ensemble déjà structuré, mais à modifier le système par " déplacement d'équilibre " jusqu'à rendre assimilable le fait inattendu ... En un mot, ce qui était perturbateur devient variation à l'intérieur d'une structure réorganisée ... Notons en outre que ces compensations de type $\beta$ prolongent en un sens les annulations de type $\alpha$... par une modification de direction également opposée. Mais ici la modification de direction nouvelle ne vise pas à annuler celle qu'introduit l'objet perturbateur : elle modifie au contraire le schème d'assimilation lui-même pour l'accommoder à l'objet et suivre son orientation.

" $\gamma$ ) La conduite de type supérieur consistera alors (ce qui est possible en toutes les situations logico-mathématiques et en certaines ex- 
plications causales bien élaborées) à anticiper les variations possibles, lesquelles perdent en tant que prévisibles et déductibles leur caractère de perturbations et viennent s'insérer dans les transformations virtuelles du système . . . Chaque transformation pouvant être entièrement annulée par son inverse ou retournée par sa réciproque, on retrouve là en un sens une situation comparable à celle d'une modification perturbatrice et de sa compensation ; seulement la grande différence ... . est que, faisant partie d'un même système dont toutes les transformations sont solidaires, l'opération $T$ implique l'existence de $T^{-1}$, ainsi que celle du produit $T \cdot T^{-1}=0:$ le sens de la compensation est par conséquent celui d'une symétrie inhérente à l'organisation du système, et non plus d'une élimination des perturbations" (pp. 71-74).

Piaget indique enfin qu'il s'agit d'une succession de phases que l'on retrouve à chacune des périodes de développement de l'enfant :

"Ce n'est pas à dire qu'il s'agisse là de trois stades généraux mais bien de phases que l'on retrouve assez régulièrement selon les domaines ou les problèmes posés au cours des périodes sensorimotrices, puis de 2 à 10-11 ans et enfin jusqu'au niveau des opérations formelles" (p. 74).

Afin que ce concept d'équilibration ne demeure pas théorique, nous l'illustrerons par un exemple de développement de notion, étudié ces dernières années à l'École de Psychologie de l'U. niversité Laval à Québec, qui nous a conduit à une illustration de développement en termes d'interaction sujet-objet.

\section{B. ILLUSTRATION DU PROCESSUS D'ÉQUILIBRATION DANS LA CONSTRUCTION DE LA NOTION DE PRO- PORTION CHEZ L'ENFANT ET L'ADOLESCENT}

\section{L'ÉPREUVE DES CONCENTRATIONS (Forme A) '}

Une expérience a été mise sur pied où le nombre rationnel est illustré concrètement, sous forme d'un ensemble de verres contenant du liquide, divisé en deux sous-ensembles de verres de jus d'orange et d'eau. La relation entre les cardinalités des deux sousensembles constitue un rapport (ou ratio), le " goût " du mélange

1. Cette recherche a été subventionnée par le ministère de l'Éducation du Québec dans le cadre des subventions d'équipe FCAC. Ce chapitre fait partie d'un ouvrage en voie d'achèvement. 
résultant étant considéré, théoriquement, directement proportionnel au nombre de verres de jus d'orange et inversement proportionnel au nombre de verres d'eau. L'expérience a été appelée épreuve des Concentrations.

Nous allons décrire, aussi sommairement que possible, les données de l'expérience effectuée.

\section{a) Instrument}

L'épreuve collective des Concentrations (forme A ou 23 items) a été mise au point pour étudier le développement de la notion de proportion. Chaque item consiste en la comparaison de deux rapports et constitue un problème de difficulté variable. Les directives sont d'abord données collectivement, avec un matériel concret et discussion en groupe de deux items de démonstration. Puis le sujet doit résoudre individuellement les 23 items expérimentaux sur sa feuille de réponse.

Le problème consiste à comparer le goût relatif de deux ensembles de liquides, composés chacun de deux sous-ensembles de verres de jus d'orange et de verres d'eau. On demande à l'enfant de choisir entre trois réponses possibles (goût plus fort à gauche, à droite, même goût), puis de justifier sa réponse par écrit, en ses propres mots, sur trois lignes figurant au bas de chaque item. Chaque item comportait ainsi une partie objective, qui a servi à l'analyse quantitative des données et l'établissement de stades de difficulté, et une partie qualitative, qui a permis d'étudier les stratégies de résolution de problème à chaque niveau.

La liste des 23 items de l'épreuve, ainsi que des deux items de démonstration I et II, figure au Tableau 1, où l'on a présenté (en notation de couple), chaque problème sous forme de deux rapports terme à terme, ou ratios, à comparer, avec la réponse correcte pour chacun, $A$ indiquant le premier ensemble, $B$ le second. Le couple correspond à la cardinalité des sous-ensembles de chaque ensemble. Le premier terme de chaque couple correspond à un nombre de verres de jus d'orange, le deuxième à un nombre de verres d'eau.

b) Échantillon

L'échantillon comprenait 321 sujets des deux sexes (151 garçons et 170 filles), dont l'âge variait entre 6 et 16 ans. Il prove- 
nait de 11 classes (une par niveau de l'élémentaire et du secondaire) d'une Régionale de la banlieue de la ville de Québec, de niveau socio-économique relativement aisé.

\section{TABLEAU 1}

Items de l'épreuve des Concentrations A (forme 23 items) numérotés selon l'ordre de présentation

\begin{tabular}{rlc}
\hline Items & Composition & $\begin{array}{c}\text { Réponse } \\
\text { correcte }\end{array}$ \\
\hline I & $(3,1)$ vs. $(1,3)$ & $A$ \\
II & $(1,1)$ vs. $(1,4)$ & $A$ \\
1 & $(1,0)$ vs. $(1,1)$ & $A$ \\
2 & $(4,1)$ vs. $(1,4)$ & $A$ \\
3 & $(1,2)$ vs. $(1,5)$ & $A$ \\
4 & $(1,2)$ vs. $(2,1)$ & $B$ \\
5 & $(1,1)$ vs. $(1,2)$ & $A$ \\
6 & $(3,1)$ vs. $(2,2)$ & $A$ \\
7 & $(1,1)$ vs. $(2,2)$ & $=$ \\
8 & $(2,3)$ vs. $(1,1)$ & $B$ \\
9 & $(2,2)$ vs. $(3,3)$ & $A$ \\
10 & $(2,2)$ vs. $(3,4)$ & $=$ \\
11 & $(1,1)$ vs. $(3,3)$ & $=$ \\
12 & $(1,2)$ vs. $(2,4)$ & $A$ \\
13 & $(2,1)$ vs. $(3,3)$ & $=$ \\
14 & $(2,3)$ vs. $(1,2)$ & $A$ \\
15 & $(4,2)$ vs. $(2,1)$ & $B$ \\
16 & $(2,1)$ vs. $(4,3)$ & $A$ \\
17 & $(1,3)$ vs. $(2,5)$ & $B$ \\
18 & $(2,1)$ vs. $(3,2)$ & $B$ \\
19 & $(2,3)$ vs. $(3,4)$ & $A$ \\
20 & $(6,3)$ vs. $(5,2)$ & $A$ \\
21 & $(3,2)$ vs. $(4,3)$ & $A$ \\
22 & $(4,2)$ vs. $(5,3)$ & \\
23 & $(5,2)$ vs. $(7,3)$ & \\
\hline
\end{tabular}

\section{c) Résultats}

Traitement quantitatif: Les résultats ont été soumis à un traitement par scalogramme. Le programme par ordinateur, portant le numéro BMD05S Guttman Scale \#1 (Dixon, 1971), a été utilisé dans l'analyse des résultats. Ce programme est basé sur la technique de Cornell, décrite par Guttman (1947). A la suite du traitement, les items sont ordonnés selon leur fréquence totale décroissante de réussite. Le scalogramme a cette particularité de 
présenter, de gauche à droite, les items en ordre décroissant de réussite, de haut en bas, les sujets en ordre croissant de succès.

Le calcul de coefficient de reproductibilité CR pour la totalité de l'échantillon est de 0.942 , alors que le coefficient de reproductibilité marginale minimum MMR est de 0.781 . On constate que certains items sont trop faciles pour l'ensemble de l'échantillon, d'autres trop difficiles. Aussi, pour valider l'échelle, nous avons recours à un coefficient qui est moins affecté par la difficulté des items, l'indice de Jackson ou PPR. Dans notre cas:

$$
\mathrm{PPR}=\frac{\mathrm{CR}-\mathrm{MMR}}{1-\mathrm{MMR}}=\frac{0.942-0.781}{1-0.781}=0.735
$$

La présente valeur s'avère suffisante, étant supérieure au seuil de 0.70 suggéré par Jackson (White et Saltz, 1957). On peut considérer l'échelle obtenue comme une échelle hiérarchique parfaite. La réussite d'un item donné implique la réussite de tous les précédents.

L'ordre de difficulté des items, ainsi que leur composition et leurs fréquences de réussite dans l'échantillon complet, sont donnés au Tableau 2.

Traitement qualitatif : Ayant vérifié le caractère hiérarchique des items, il s'agissait d'étudier s'il existait un regroupement possible des items en catégories définies selon un critère algébrique.

Une première catégorisation permet de découper la succession des 23 items, ordonnés en ordre décroissant de réussite, en trois catégories génériques distinctes. Elle figure au Tableau 2 sous la forme des catégories génériques I, II et III. La catégorie I correspond aux problèmes nécessitant une comparaison de.termes. Elle se subdivise en catégories spécifiques IA : nécessitant une comparaison des premiers termes de chaque couple ; IB : nécessitant une comparaison des deuxièmes termes de chaque couple ; IC : impliquant une confrontation des deux termes à l'intérieur de chaque couple.

La catégorie II correspond aux classes d'équivalence de rapports, subdivisée en IIA où les termes sont égaux, IIB où ils sont 


\section{TABLEAU 2}

Fréquences totales des réussites des items telles qu'attribuées par le scalogramme, avec catégorisation ultérieure des items

\begin{tabular}{llll} 
Catégo- & Catégo- & \multicolumn{1}{c}{$\begin{array}{l}\text { Fréquence des } \\
\text { réussites at- } \\
\text { tribuées par }\end{array}$} & $\begin{array}{l}\text { Caracté- } \\
\text { ries gé- } \\
\text { nériques spé- }\end{array}$ cifiques Items \\
& Composition & $\begin{array}{l}\text { le scalogramme } \\
\text { ristiques }\end{array}$
\end{tabular}

\begin{tabular}{|c|c|c|c|c|}
\hline 0 & 0 & 0 & & 321 \\
\hline \multirow[t]{3}{*}{ I } & IA & $\begin{array}{l}2 \\
6 \\
4\end{array}$ & $\begin{array}{l}(4,1) \text { vs. }(1,4) \\
(3,1) \text { vs. }(2,2) \\
(1,2) \text { vs. }(2,1)\end{array}$ & $\begin{array}{l}319 \text { Le premier terme d'un } \\
319 \text { rapport prédomine autant } \\
319 \text { à l'intérieur du rapport } \\
\text { qu'entre les rapports.* }\end{array}$ \\
\hline & IB & $\begin{array}{l}1 \\
3 \\
5\end{array}$ & $\begin{array}{l}(1,0) \text { vs. }(1,1) \\
(1,2) \text { vs. }(1,5) \\
(1,1) \text { vs. }(1,2)\end{array}$ & $\begin{array}{l}311 \text { A égalité de premiers } \\
307 \text { termes, différences entre } \\
305 \text { deuxièmes termes des } \\
\text { rapports }\end{array}$ \\
\hline & IC & $\begin{array}{r}8 \\
13 \\
10\end{array}$ & $\begin{array}{l}(2,3) \text { vs. }(1,1) \\
(2,1) \text { vs. }(3,3) \\
(2,2) \text { vs. }(3,4)\end{array}$ & $\begin{array}{l}295 \text { Egalité de termes dans } \\
291 \text { un rapport, différence } \\
297 \text { dans l'autre. }\end{array}$ \\
\hline \multirow[t]{2}{*}{ II } & IIA & $\begin{array}{r}9 \\
11 \\
7\end{array}$ & $\begin{array}{l}(2,2) \text { vs. }(3,3) \\
(1,1) \text { vs. }(3,3) \\
(1,1) \text { vs. }(2,2)\end{array}$ & $\begin{array}{l}251 \text { Équivalence de rapports } \\
244 \text { avec égalité des termes } \\
231 \text { de chaque rapport. }\end{array}$ \\
\hline & IIB & $\begin{array}{l}12 \\
15\end{array}$ & $\begin{array}{l}(1,2) \text { vs. }(2,4) \\
(4,2) \text { vs. }(2,1)\end{array}$ & $\begin{array}{l}186 \text { Equivalence des rapports } \\
156 \text { avec différence des ter- } \\
\text { mes de chaque rapport. }\end{array}$ \\
\hline \multirow[t]{3}{*}{ III } & III A1 & $\begin{array}{l}16 \\
17 \\
14 \\
18\end{array}$ & $\begin{array}{l}(2,1) \text { vs. }(4,3) \\
(1,3) \text { vs. }(2,5) \\
(2,3) \text { vs. }(1,2) \\
(2,1) \text { vs. }(3,2)\end{array}$ & $\begin{array}{l}141 \text { Inégalité des rapports } \\
131 \text { avec deux termes corres- } \\
107 \text { pondants multiples l'un } \\
88 \text { de l'autre. }\end{array}$ \\
\hline & IIIA2 & $\begin{array}{l}20 \\
22 \\
19 \\
21\end{array}$ & $\begin{array}{l}(6,3) \text { vs. }(5,2) \\
(4,2) \text { vs. }(5,3) \\
(2,3) \text { vs. }(3,4) \\
(3,2) \text { vs. }(4,3)\end{array}$ & $\begin{array}{l}87 \text { Inégalité des rapports } \\
71 \text { avec simplification pos- } \\
65 \text { sible ou extraction } \\
59 \text { de l'unité }(1,1) \text {. }\end{array}$ \\
\hline & IIIB & 23 & $(5,2)$ vs. $(7,3)$ & $\begin{array}{l}51 \text { Inégalité des rapports } \\
\text { avec termes correspon- } \\
\text { dants premiers entre eux. }\end{array}$ \\
\hline
\end{tabular}

Note- Nombre total de sujets de l'échantillon $\mathrm{N}=321$.

*Lors de la présentatión "standardisée " des items. 
inégaux. Enfin la catégorie III correspond aux problèmes nécessitant le recours à un dénominateur commun.

En vue d'une étude de l'aspect chronologique de la présente échelle de catégories, le Tableau 3 présente la distribution des sujets en fréquences, en fonction de l'âge et des catégories spécifiques dans lesquelles ils se situent. Les données de ce tableau permettent de calculer l'âge médian des sujets réunis dans chacun des groupes, ainsi que le seuil de signification des différences observées entre la distribution d'âge des sujets de chaque groupe et ceux du groupe précédent, selon le test Kolmogorov-Smirnov. On constate une différence significative entre les stades IC, IIA, IIB, IIIA et IIIB.

On a ajouté au Tableau 3 les âges d'accession à chacun de ces stades, soit l'âge où $50 \%$ des sujets ont atteint le stade. On constatera une différence notable entre les âges médians, qui correspondent aux sujets situés au stade même, et les âges d'accession, qui tiennent compte des sujets ayant passé à des stades supérieurs.

Afin de différencier les stades inférieurs de l'épreuve, on a mis au point une formule individuelle (Forme $B$ ), qui a permis de différencier significativement les stades IB + IC du stade IA (Noelting et al., en préparation).

\section{STRATÉGIE DE RÉSOLUTION DE PROBLÈME ET SYMBOLISME UTILISÉ}

\section{a) Les composantes d'un item.}

Afin d'analyser les justifications présentées par l'enfant aux divers items tant ratés que réussis, on a mis au point un symbolisme permettant de transcrire les relations et opérations effectuées par les sujets à chaque item.

Chacun des deux mélanges à comparer, composé d'un certain nombre de verres de jus d'orange et d'un certain nombre de verres d'eau, peut être considéré comme un ensemble composé de deux sous-ensembles complémentaires : un sous-ensemble protagoniste, constitué de verres de jus d'orange, un sous-ensemble antagoniste, composé de verres d'eau. 


\section{TABLEAU 3}

Répartition des sujets en fréquences, suivant l'âge et les catégories d'items réussis de l'épreuve des Concentrations $A$

\begin{tabular}{|c|c|c|c|c|c|c|c|c|c|}
\hline \multirow{3}{*}{ Âges } & \multirow{3}{*}{$\mathbf{N}$} & \multicolumn{8}{|c|}{ Catégories génériques et spécifiques } \\
\hline & & \multicolumn{4}{|c|}{ I } & \multicolumn{2}{|r|}{ II } & \multicolumn{2}{|r|}{ III } \\
\hline & & 0 & IA & IB & IC & IIA & IIB & IIIA & IIIB \\
\hline 6 & 14 & 0 & 1 & 2 & 8 & 3 & 0 & 0 & 0 \\
\hline 7 & 26 & 1 & 1 & 7 & 14 & 2 & 1 & 0 & 0 \\
\hline 8 & 35 & 1 & 0 & 4 & 12 & 10 & 6 & 2 & 0 \\
\hline 9 & 43 & 0 & 1 & 2 & 9 & 12 & 13 & 6 & 0 \\
\hline 10 & 32 & 0 & 0 & 1 & 3 & 13 & 8 & 6 & 1 \\
\hline 11 & 38 & 0 & 0 & 1 & 5 & 12 & 7 & 9 & 4 \\
\hline 12 & 34 & 0 & 3 & 1 & 0 & 9 & 5 & 14 & 2 \\
\hline 13 & 31 & 0 & 2 & 0 & 0 & 2 & 9 & 17 & 1 \\
\hline 14 & 20 & 0 & 0 & 0 & 1 & 1 & 2 & 10 & 6 \\
\hline 15 & 29 & 0 & 0 & 0 & 0 & 0 & 8 & 16 & 5 \\
\hline 16 & 19 & 0 & 0 & 0 & 0 & 1 & 2 & 8 & 8 \\
\hline \multicolumn{2}{|c|}{ Total 321} & 2 & 8 & 18 & 52 & 65 & 61 & 88 & 27 \\
\hline \multicolumn{2}{|c|}{ Âge médian } & - & - & - & $7 ; 8$ & $10 ; 4$ & $11 ; 11$ & $13 ; 5$ & $14 ; 9$ \\
\hline \multicolumn{2}{|c|}{$p^{2}$} & - & - & - & - & $<.01$ & $<.01$ & $<.01$ & $<.01$ \\
\hline \multicolumn{2}{|c|}{ Âges d'accession 3} & - & - & - & - & $8 ; 1$ & $10 ; 5$ & $12 ; 2$ & $(17 ; 0)$ \\
\hline
\end{tabular}

Notes.

1. Les âges indiqués sont les âges médians de chaque distribution, une fois les fréquences cumulées et transformées en pourcentages.

2. Seuil de signification des différences observées entre la distribution du stade et celle du stade précédent, selon le test Kolmogorov-Smirnov.

3. Les âges d'accession correspondent à l'âge où $50 \%$ des sujets de l'âge réussissent au moins un item du stade.

La notion impliquée dans le problème est le "goût " du mélange, soit un rapport entre le nombre de verres de jus et le nombre de verres d'eau dans l'ensemble. Il s'agit en l'occurrence d'un rapport de deux quantités, que l'on pourrait appeler un " ratio "; ce n'est qu'au stade formel supérieur, vers 14-15 ans, que l'adolescent, dans la situation donnée, passera spontanément à des rapports de parties à tout ou " fractions ", rapportant le nombre de verres de jus d'orange au nombre total de verres de liquide. 
Les cardinalités des deux sous-ensembles complémentaires d'un ensemble peuvent être considérées comme formant un couple ordonné $(a, b)$, le premier terme correspondant au nombre de verres de jus d'orange, le deuxième terme au nombre de verres d'eau, du mélange. Il s'agit bien d'un couple ordonné, puisque les deux termes ne sont pas interchangeables: $(a, b)$ n'est pas équiva. lent à $(b, a)$.

Les deux termes $a$ et $c$, ou premiers termes des couples, correspondent à la cardinalité des deux sous-ensembles protagonistes, ou nombre de verres de jus d'orange de chaque ensemble. Les deux termes $b$ et $d$, ou deuxièmes termes des couples, correspondent à la cardinalité des deux sous-ensembles antagonistes, ou nombre de verres d'eau dans chaque ensemble. On dira que les deux termes $a$ et $b$, ou $c$ et $d$, d'un couple, sont les termes complémentaires d'un couple ; les deux termes $a$ et $c$, ou $b$ et $d$, seront dits termes correspondants d'un couple à l'autre.

b) Symboles algébriques utilisés pour traduire un item et ses composantes.

Appelons $A$ et $B$ les deux ensembles à comparer. Appelons $u$ et $v$ les cardinalités respectives des deux ensembles $A$ et $B, a$ et $b$ les cardinalités respectives des sous-ensembles complémentaires de l'ensemble $A, c$ et $d$ celles des sous-ensembles complémentaires de l'ensemble $B$. L'enfant procédera à la comparaison de deux ratios $a: b$ vs. $c: d$, que nous pouvons traduire sous forme de deux couples $(a, b)$ vs. $(c, d)$.

c) Présentation standardisée d'un item.

Afin de trouver la structure commune des items d'un même stade, il est bon de présenter les deux couples qui composent l'item selon une règle commune. C'est ainsi que l'on présentera, à gauche, le couple ayant le plus grand premier terme, ou, à égalité de premiers termes, à droite le couple à plus grand deuxième terme.

d) Les deux modes $E$ et I de résolution d'un problème de rapport.

À chacun des stades trouvés, nous pouvons isoler deux variantes fonctionnelles de résolution de problème : 
i) Le mode $\mathrm{E}$ (ou intercouple), où le sujet procède par covariation, ou variation conjointe, des deux termes d'un couple, pour retrouver l'autre couple. Ce mode aboutit au stade IIIB à l'algorithme du dénominateur commun.

ii) Le mode I (ou intracouple), où le sujet isole d'abord le rapport entre termes à l'intérieur d'un couple, pour le comparer au rapport entre termes dans l'autre couple. Ce mode aboutit au stade IIIB à l'algorithme du pourcentage.

e) Formalisation des stratégies de résolution caractéristiques de chaque stade.

Au Tableau 4, on trouvera les stratégies de résolution caractéristiques de chaque stade (en utilisant le symbolisme qui vient d'être présenté), permettant de résoudre tous les items du stade, et illustrées par un item typique du stade. On trouvera plus loin quelques-uns parmi les exemples individuels de réussite, avec explication, qui nous ont permis de dégager ces stratégies. On a défini les stades d'après les comportements typiques que l'on y a trouvés, selon la chronologie de développement en périodes, stades et sous-stades de Piaget. Cependant les divers sous-stades (IA, IB . . IIA, IIB, etc.) deviennent ici des stades véritables, puisqu'ils se différencient tant par la chronologie que par la structure. Nous obtenons ainsi une succession de buit stades différenciés par leur structure.

3. ANALYSE DES STRATÉgIES AUX DEUX PÉRIODES SUCCESSIVES DE L'ÉLABORATION DU RAISONNEMENT PRO. PORTIONNEL.

a) Les deux périodes d'équilibration.

L'épreuve des Concentrations présente une illustration concrète de la notion de rapport. En l'étudiant génétiquement, nous pouvons décrire le processus d'élaboration du raisonnement proportionnel à deux périodes successives de développement chez l'enfant et l'adolescent. 


\section{TABLEAU 4}

\section{Stratégies appliquées pour résoudre les problèmes aux différents stades de développement de la notion de rapport}

\begin{tabular}{|c|c|c|c|c|}
\hline \multicolumn{2}{|c|}{$\begin{array}{l}\text { Périodes Dénomina- } \\
\text { \& stades tions }\end{array}$} & \multicolumn{2}{|c|}{$\begin{array}{ll}\text { Âges d'ac- } & \text { Items typiques } \\
\text { cession }(50 \% \text { ss }) & (a, b) \text { vs. }(c, d) \\
\end{array}$} & \multirow{2}{*}{$\begin{array}{l}\text { Stratégies ca- } \\
\text { ractéristiques }\end{array}$} \\
\hline 0 & Symbolique & $(2 ; 0)$ & $(1,0)$ vs. $(0,1)$ & \\
\hline IA & $\begin{array}{l}\text { Intuitif } \\
\text { inférieur }\end{array}$ & $(3 ; 6)$ & $(4,1)$ vs. $(1,4)$ & $\begin{array}{c}a>c \\
\operatorname{donc}(a, b)>(c, d)\end{array}$ \\
\hline IB & $\begin{array}{l}\text { Intuitif } \\
\text { moyen }\end{array}$ & $6 ; 4$ & $(1,2)$ vs. $(1,5)$ & $\begin{array}{l}a=c, b<d \\
\operatorname{donc}(a, b)>(c, d)\end{array}$ \\
\hline IC & $\begin{array}{l}\text { Intuitif } \\
\text { supérieur }\end{array}$ & $7 ; 0$ & $(3,4)$ vs. $(2,1)$ & $\begin{array}{l}a<b, c>d \\
\text { donc }(a, b)<(c, d)\end{array}$ \\
\hline IIA & $\begin{array}{l}\text { Opératoire } \\
\text { concret in- } \\
\text { férieur }\end{array}$ & $8 ; 1$ & $(1,1)$ vs. $(2,2)$ & $m(1,1)=(m, m)$ \\
\hline IIB & $\begin{array}{l}\text { Opératoire } \\
\text { concret su- } \\
\text { périeur }\end{array}$ & $10 ; 5$ & $(2,3)$ vs. $(4,6)$ & $\begin{array}{l}m(a, b)=(m a, m b) \\
\text { avec } a \neq b\end{array}$ \\
\hline IIIA & $\begin{array}{l}\text { Opératoire } \\
\text { formel in- } \\
\text { férieur }\end{array}$ & $12 ; 2$ & $(3,1)$ vs. $(5,2)$ & $\begin{array}{l}m b=d \\
m(a, b)=(m a, m b)=(m a, d) \\
(m a, d)>(c, d) \\
\operatorname{donc}(a, b)>(c, d)\end{array}$ \\
\hline IIIB & $\begin{array}{l}\text { Opératoire } \\
\text { formel su- } \\
\text { périeur }\end{array}$ & $15 ; 10$ & $(3,5)$ vs. $(5,8)$ & $\begin{array}{l}a+b=u ;(a, b) \rightarrow(a, u) \\
c+d=v ;(c, d) \rightarrow(c, v) \\
u v=v u \\
u(c, v)=(u c, u v) \\
v(a, u)=(v a, v u) \\
(u c, u v)>(v a, v u) \\
\text { donc }(c, d)>(a, b)\end{array}$ \\
\hline
\end{tabular}

NOTE - Certains items typiques proviennent de formes différentes de l'épreuve.

La période d'équilibration I (préopératoire) correspond aux stades IA, IB, IC et IIA de l'épreuve des Concentrations. L'on assiste à la construction progressive de la notion de rapport $1: 1$, que nous traduisons sous la forme de couple $(1,1)$, par articulation de la variation d'un terme direct (ler terme du couple) avec la variation d'un terme inverse (2ème terme), pour constituer un inva- 
riant de transformation qui est le couple $(1,1)$. Il y a " covariation" possible des deux termes de ce couple, pour donner la "classe d'équivalence " du couple. On peut décrire logiquement la covariation comme une variation biconditionnelle ou équivalente des deux termes du couple, soit $(p \leftrightarrows q)$, où $p$ et $q$ représentent, respectivement, la variation multiplicative de chacun des termes du couple.

La période d'équilibration II (opératoire concrète et formelle) correspond aux stades IIA, IIB, IIIA et IIIB de l'épreuve des Concentrations. L'opération logique d'équivalence $(p \stackrel{\leftrightarrows}{\leftrightarrows})$, ou covariation des deux termes du couple à l'intérieur de sa classe d'équivalence, s'articule progressivement avec l'opération logique de disjonction exclusive $(r \mathrm{~W} s)$, ou variation singulière d'un terme avec constance de l'autre, pour constituer les divers algorithmes du dénominateur commun et des opérations sur les rationnels. Nous représentons par $r$ et $s$, respectivement, les variations additives des deux termes d'un couple.

Les deux périodes successives d'équilibration sont décrites ciaprès, avec des exemples caractéristiques de comportements observés chez l'enfant. $\AA$ chaque stade on a donné un exemple de réussite, avec les explications fournies par l'enfant, et un exemple d'échec à un item du stade suivant, ce qui permettra ultérieurement d'abstraire les mécanismes de passage de l'échec à la réussite, lors d'un changement de stade.

Comme on le constatera, nous mettons en évidence, à chaque période d'équilibration, quatre phases successives, que nous dénommons, $a, b, c, d$.

Les deux premières phases $a$ et $b$ correspondent à la dualité des conduites que distingue Piaget : la première lorsqu'il y a élimination de la perturbation, la deuxième lorsqu'il y a modification du schème pour " compenser " la perturbation. C'est ainsi que nous interprétons une "perturbation" comme une modification (ou nouveauté) survenue dans l'objet familier d'un schème. La phase a correspondra à l'équilibre entre schème et partie familière de l'objet, avec élimination de la perturbation considérée comme étrangère à l'échange. La phase $b$ correspondra à une modification $\mathrm{du}$ schème pour " compenser " la perturbation dans l'objet, avec assimilation de la partie perturbatrice de l'objet à côté de la partie 
familière. La phase $c$ correspondra à une coordination entre partie familière et partie perturbatrice de l'objet. Enfin la phase $d$ consiste en une intégration biérarchique des deux parties familière et perturbatrice de l'objet dans un système de transformations de l'objet et de ses parties.

b) Période d'équilibration I: préopératoire (4-5 à 8.9 ans)

Les quatre phases dans la combinaison d'une variation directe de quantité avec une variation inverse, pour construire la notion de couple $(1,1)$ et de sa classe d'équivalence.

Cette construction s'effectuera en quatre étapes que nous allons examiner en détail. Les items numérotés, précédés de la lettre $A$, proviennent de la forme collective $A$ de l'épreuve ; ceux précédés de la lettre $\mathrm{B}$, proviennent de la forme individuelle $\mathrm{B}$.

Problème : Soit deux ensembles à comparer, composés chacun de deux sous-ensembles consistant en un certain nombre de verres de jus d'orange et d'eau. On représente les sous-ensembles par leur cardinalité au moyen des deux couples $(a, b)$ et $(c, d)$. Le problème consiste à déterminer le rapport entre les cardinalités des sous-ensembles à l'intérieur de chaque ensemble, pour en déterminer le plus grand.

Phase $a$. - Stade IA : Centration sur les premiers termes des couples. Réussite des items de type $(4,1)$ vs. $(1,4)$ ou $(2,1)$ vs. $(1,2)$.

Exemple :

Diane, 4;0 B4: (1,2) vs. (2,1) «C'est ici (B) qu'il y a Choisit $B$ (réussite). le plus d'orange".

Le sujet est capable d'effectuer une comparaison de grandeur entre les premiers termes des deux couples. Mis en présence des deuxièmes termes, le sujet les ignore simplement ou les juxtapose comme éléments différents. Avec l'item illustré, présenté sous sa forme standardisée $(2,1)$ vs. $(1,2)$, la stratégie mise en jeu est la suivante :

$$
a>c, \text { donc }(a, b)>(c, d)
$$

Devant un item comme $(1,1)$ vs. $(1,0)$ de stade $I B$, le sujet échoue. 
Exemple :

Louis, $4 ; 7$

D6 : $(1,1)$ vs. $(1,0)$ " Ca va goûter pareil Choisit $=$ (échec). parce qu'il y a 1 verre d'orangeade là (B) et 1 verre d'orangeade là (A)".

Il choisit l'égalité, puisqu'il ne considère pas les deuxièmes termes des couples.

Phase $b$. - Stade IB : Concordance des premiers termes, différence des deuxièmes termes des couples. Réussite des items de type $(1,0)$ vs. $(1,1)$ ou $(1,2)$ vs. $(1,5)$.

Exemple :

Sylvain, $4 ; 7$

D6 : $(1,1)$ vs. $(1,0)$ "Chez moi (B) sa va Choisit $B$ (réussite). goûter plus l'orange et pis chez toi $(A)$ ça va goûter un petit peu l'orange".

Le sujet est maintenant capable de considérer les deuxièmes termes des couples comme ayant un effet inverse aux premiers termes sur le couple total. Cela permet d'établir une relation entre les deuxièmes termes des couples lors d'une constance des premiers termes. Avec l'item illustré, présenté sous sa forme standardisée : $(1,0)$ vs. $(1,1)$, la stratégie mise en jeu est la suivante:

$$
a=c, b<d \text {, donc }(a, b)>(c, d)
$$

Cependant, devant un item comme $(3,4)$ vs. $(2,1)$ de stade IC, le sujet échoue.

Exemple :

Nathalie, 6;0 $\quad$ B9: $(2,1)$ vs. $(3,4)$ " En $B$, il $y$ a 3 verres Choisit $B$ (échec). d'orange. C'est plus qu'en $A$ ".

Le sujet ne peut comparer deux relations de différence, et régresse à la centration sur le jus d'orange. 
Phase c. - Stade IC: Confrontation de deux relations entre termes complémentaires des deux couples. Réussite des items de type $(2,3)$ vs. $(1,1)$ ou $(3,4)$ vs. $(2,1)$.

Exemples :

François, 6;0

B8 : (2,3) vs. $(1,1)$ «En $B$, on a 1 verre de Choisit $B$ (réussite). jus, 1 verre d'eau; en $A$, on a 2 verres de jus et 3 verres d'eau. Il y a trop le goût de l'eau en $A$ ».

Guy, 7;0

B9 : $(2,1)$ vs. $(3,4)$ "En $A$, pour 2 verres Choisit $A$ (réussite). de jus on a 1 verre d'eau seulement, alors qu'en $B$, on a 3 verres de jus pour 4 verres d'eau".

Le sujet est maintenant capable d'établir une relation entre les deux termes d'un couple, et de la confronter avec la relation entre les deux termes de l'autre couple. La stratégie mise en jeu est la suivante :

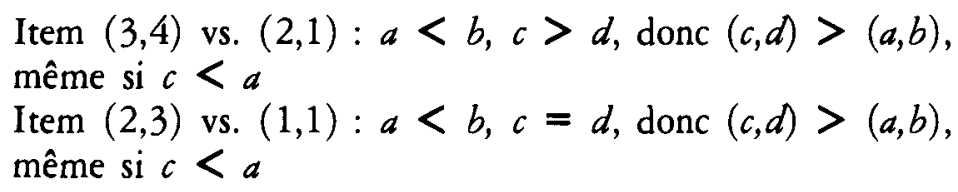
même si $c<a$

Item $(2,3)$ vs. $(1,1): a<b, c=d$, donc $(c, d)>(a, b)$, même si $c<a$

Cependant un item comme $(1,1)$ vs. $(2,2)$, de stade IIA, est échoué.

Exemples :

Éric, 6;0

B12: (2,2) vs. $(3,3)$ "Parce qu'il y a plus Choisit $B$ (échec). d'orange en $B$ ".

Nadia, 5;0

B11: (1,1) vs. $(2,2)$ "Parce qu'en $A$ il y a Choisit $A$ (échec). juste un verre d'eau et ga va goûter plus le jus ». 
Le sujet est incapable de multiplier une égalité entre termes complémentaires à l'intérieur des couples par une variation entre termes correspondants des couples. Le sujet régresse à une centration, soit sur les verres de jus, soit sur les verres d'eau.

Phase $d$. - Stade IIA : Schème de covariation $(1,1)$. Réussite des items de type $(2,2)$ vs. $(1,1)$ ou $(3,3)$ vs. $(2,2)$.

Exemples :

Mode I (intracouple)

Martine, 8;0

B12: (2,2) vs. $(3,3)$ " 2 pour 2 en $A ; 3$ Choisit = (réussite). pour 3 en $B$ ».

Mode $\mathrm{E}$ (intercouple)

Johanne, $11 ; 0$

A7 : $(1,1)$ vs $(2,2)$ "Chaque verre dilue Choisit $=$ (réussite). un verre. Alors $A$ a un verre de jus et $B 2$ verres; $A$ a un verre d'eau et $B$ deux. Il reste une égalité, seulement il $y$ a plus de liquide mélangé dans $B$ m.

Le sujet différencie la relation entre termes complémentaires à l'intérieur du couple (stabilisée sous forme de rapport invariant) et leur covariation en grandeur (mobilisée sous forme de " complexification" ou "simplification" du couple), pour constituer la classe d'équivalence la plus simple, la classe d'équivalence $(1,1)$. La classe d'équivalence résulte d'une différenciation entre invariant et transformation. La stratégie mise en jeu est la suivante :

$$
\begin{array}{ll} 
& \text { Mode E (intercouple) } \\
& m(1,1)=(m, m) \\
\text { avec } & (1,1) \simeq(m, m) \\
& \text { Mode I (intracouple) } \\
& m / m=n / n \\
\text { même } & \text { si } m \neq n
\end{array}
$$

Il y a constitution de la notion d'équivalence $\simeq$, qui est une relation " générique " caractérisant la classe d'équivalence, distincte de la 
notion d'égalité $=$, qui est une relation "spécifique " entre éléments d'une classe d'équivalence.

$$
\text { Ainsi } \begin{aligned}
(1,1) & =(1,1) \\
& m(1,1)=(m, m) \\
\text { mais } \quad(1,1) & \simeq(m, m)
\end{aligned}
$$

La covariation multiplicative $m(1,1)=(m, m)$ correspond à une " complexification" du couple $(1,1)$.

De même $(m, m)=m(1,1)$

$$
\text { mais }(m, m) \simeq(1,1)
$$

La covariation inverse $(m, m) / m=(1,1)$ correspond à une " simplification"du couple $(m, m)$. Tous deux correspondent à des covariations des deux termes du couple.

Du point de vue logique, la constitution d'une classe d'équivalence correspond à une covariation ou équivalence dans la variation des deux termes du couple. Illustrons cela en interprétant la transformation sur un terme d'un couple comme une "proposition ".

$$
\text { Mode E (intercouple) }
$$

Exemple :

$$
(1,1) \text { vs. }(2,2) \text {. Dans ce cas : }(a, b)=(1,1) \text {. }
$$

Appelons $p: m . a=m a=c$

$$
q: m \cdot b=m b=d
$$

L'équivalence ( $p \longleftrightarrow q$ ) définit la " complexification " d'un couple. La "simplification" d'un couple correspond aux mêmes transformations appliquées dans le sens inverse :

$$
\begin{aligned}
& p^{\prime}: c / m=a \\
& q^{\prime}: d / m=b
\end{aligned}
$$

où $m$ est un nombre naturel (à l'exclusion de 1).

Nous aurons la négation de ces transformations :

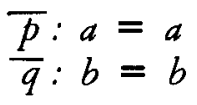

La classe d'équivalence est constituée par la variation conjointe, par multiplication ou division, des deux termes du couple. La réso- 
lution d'un item comme $(3,3)$ vs. $(2,2)$ demande la simplification préalable d'un couple, puis sa complexification, lors du passage à l'autre couple.

\section{Mode I : (intracouple)}

Cependant l'enfant résout également le problème par le mode intracouple en procédant à une division des termes à l'intérieur de chaque couple, pour montrer l'égalité des deux quotients obtenus.

Isolons les opérations élémentaires de ces stratégies que nous interprétons comme des "propositions " et auxquelles nous appliquons les règles de la logique des propositions. Un certain nombre de théorèmes sont nécessaires, sur lesquels nous n'insisterons pas ici, pour passer de $p$ : opération à $p$ : proposition.

$$
\text { Appelons } \begin{aligned}
p: m \cdot a & =m a=b \\
q: m \cdot c & =m c=d
\end{aligned}
$$

où $m$ est un nombre naturel (à l'exclusion de 1).

Nions ces propositions :

$$
\begin{aligned}
& \bar{p}: a=a \\
& \bar{q}: c=c
\end{aligned}
$$

La classe d'équivalence résulte de transformations équivalentes appliquées aux termes complémentaires d'un couple. La table de vérité de l'équivalence s'établit comme suit :

\begin{tabular}{cc|cc}
\hline$p$ & $q$ & $p$ & $\leftrightarrows$ \\
1 & 1 & & 1 \\
1 & 0 & & 0 \\
0 & 1 & & 0 \\
0 & 0 & & 1 \\
\hline
\end{tabular}

En effet $p . q$ est vrai ainsi que $\bar{p} \cdot \bar{q}$. pour caractériser l'équivalence.

Cette période d'équilibration se termine par la constitution de la classe d'équivalence de rapport la plus simple, la classe d'équivalence $(1,1)$. Partant d'une variation unaire $a \rightarrow c$, l'enfant a construit le schème binaire $(1,1) \rightarrow(m, m)$. La première période d'équilibration trouvée est résumée au Tableau 5 , où l'on a décrit les mécanismes de passage d'un stade à l'autre pour chacune de ses phases. 


\section{TABLEAU}

Les quatre phases de la construction de la notion de rapport, à partir du schème de variation unaire $[a>c]$, durant la période d'équilibration I (préopératoire)

\begin{tabular}{|c|c|c|c|c|c|}
\hline $\begin{array}{l}\text { Stades opéra- } \\
\text { toires piagé- } \\
\text { tiens }\end{array}$ & $\begin{array}{l}\text { Items } \\
(a, b)\end{array}$ & $\begin{array}{l}\text { Is caractéristiques } \\
\text { vs. }(c, d)\end{array}$ & Stratégies de résolution & Mécanismes d'équilibration & $\begin{array}{l}\text { Caractéristiques des phases successives d'é- } \\
\text { quilibration }\end{array}$ \\
\hline $\begin{array}{l}\quad \text { IA } \\
\text { Intuitif } \\
\text { inférieur }\end{array}$ & & $(4,1)$ vs. $(1,4)$ & $\begin{array}{c}a>c \\
\text { donc }(a, b)>(c, d) \\
\quad(\text { ou } d>b \\
\text { donc }(d, c)>(b, a)\end{array}$ & $\begin{array}{l}\text { Centration successive sur chacun des } \\
\text { termes considérés indépendamment. }\end{array}$ & $\begin{array}{l}\text { Schème de variation, des premiers termes } \\
\text { des couples, change d'objet en passant aux } \\
\text { deuxièmes termes. }\end{array}$ \\
\hline $\begin{array}{l}\text { IB } \\
\text { Intuitif } \\
\text { moyen }\end{array}$ & & $(1,2)$ vs. $(1,5)$ & $\begin{array}{l}\quad a=c, b<d \\
\text { donc }(a, b)>(c, d)\end{array}$ & $\begin{array}{l}\text { Différenciation objectale, par in- } \\
\text { version de signe du deuxième } \\
\text { terme: } b=1 / a \text {. }\end{array}$ & $\begin{array}{l}\text { Schème de variation, des premiers termes } \\
\text { des couples, change de direction en passant } \\
\text { aux deuxièmes termes. }\end{array}$ \\
\hline $\begin{array}{l}\quad \text { IC } \\
\text { Intuitif } \\
\text { supérieur }\end{array}$ & i) & $(3,4)$ vs. $(2,1)$ & $\begin{array}{l}\quad a<b, c>d \\
\text { donc }(a, b)<(c, d) \\
\text { même si } a>c\end{array}$ & $\begin{array}{l}\text { Différenciation opérative, par in- } \\
\text { version de la relation entre termes : } \\
(a R b) \text { en }\left(c R^{\prime} d\right) \text {. }\end{array}$ & $\begin{array}{l}\text { Schème de relation, entre termes d'un } \\
\text { couple, se renverse en passant à l'autre } \\
\text { couple. }\end{array}$ \\
\hline $\begin{array}{l}\text { IIA } \\
\text { Opératoire } \\
\text { concret in- } \\
\text { férieur }\end{array}$ & ii) & $(2,3)$ vs. $(1,1)$ & $\begin{array}{l}a<b, c=d \\
\text { donc }(a, b)<(c, d) \\
\text { même si } a>c \\
m(1,1)=(m, m) \\
(1,1) \simeq(m, m)\end{array}$ & $\begin{array}{l}a R b=c R d \neq a T c=b T d \\
\text { Différenciation de la relation intra- } \\
\text { couple, qui donne l'invariant, et de } \\
\text { la relation intercouples, qui donne la } \\
\text { transformation, combinées en une } \\
\text { "covariation" des } 2 \text { termes d'un } \\
\text { couple (donnant une classe d'équi- } \\
\text { valence). }\end{array}$ & $\begin{array}{l}\text { Schème de variation conjointe de termes } \\
\text { égaux, excluant les éléments excédentaires. }\end{array}$ \\
\hline
\end{tabular}


La période d'équilibration qui suit portera, contrairement à la précédente, sur des transformations binaires, avec libération des deux termes du couple l'un par rapport à l'autre, autant à l'intérieur du couple (indépendance des termes) que dans les transformations effectuées sur le couple (opération sur les rapports).

c) Période d'équilibration II: opératoire concrète et formelle (8-9 à $15-16$ ans)

Les quatre phases dans la combinaison de l'équivalence $(\mathrm{p} \leftrightarrows \mathrm{q})$ avec la disjonction exclusive $(\mathrm{r} W \mathrm{~s})$, pour constituer les algorithmes du dénominateur commun et les opérations sur les rationnels.

Le sujet au cours de la période d'équilibration précédente a construit l'invariant $(1,1)$, qu'il peut covarier en $(m, m)$ ou le rapport $\mathrm{m} / \mathrm{m}$, qu'il peut diviser en $1 / 1$, basé sur le schème logique de conjonction des termes dans la variation (stade IIA). Le problème nouveau consiste à introduire des variations arithmétiques de grandeur des termes, à l'intérieur de ce schème logique de conjonction $(1,1)$. Examinons dans le détail les quatre étapes de cette nouvelle période de construction.

Phase a. - Stade IIA : Schème de covariation $(1,1)$ ou de division $1 / 1$.

La première phase de cette nouvelle période d'équilibration correspond à la phase d'achèvement de la période précédente. Ce sont les erreurs nouvelles, effectuées par les sujets de cette phase, dans des problèmes de rapport où les composantes ne sont pas dans un rapport $1: 1$, qui provoquent la nouvelle étape d'équilibration.

Au stade IIA, il y a covariation possible des deux termes, direct et inverse, d'un rapport, lorsqu'ils sont égaux. Ceci correspond à un syncrétisme, ou une indifférenciation, entre la liaison logique de conjonction entre deux termes $(p \leftrightarrows q)$, et la grandeur aritbmétique de ces termes : $a=b$.

En présence de termes excédant le rapport $(1,1)$, le sujet les " isole " et se base sur eux pour effectuer un jugement (centration sur le reste). Ainsi la stratégie de covariation $(1,1)$ conduit à l'erreur avec un problème plus complexe comme $(1,2)$ vs. $(2,4)$.

Il s'agit bien d'une phase $a$ d'équilibration, parce que le sujet 
traite séparément la partie de l'objet adéquate au schème, (par un schème multiplicatif) et la partie excédentaire dans l'objet, (par un schème additif).

Aussi il y a échec aux items de type $(1,2)$ vs. $(2,4)$, de stade IIB, le sujet appliquant son schème inadéquat de compensation $(1,1)$ et isolant ainsi un " reste "dans le milieu, reste qui lui sert à juger de la situation, avec confusion de l'aspect " additif " du reste et "divisif " du schème $(1,1)$.

Exemples:

Louise, $11 ; 0$

A1 2 : $(1,2)$ vs. $(2,4)$ « Parce que gaucbe a Choisit $A$ (échec). un pot d'eau de plus tandis que droite en a deux de plus".

Diane, $12 ; 0$

A12: (1,2) vs. $(2,4)$ "Dans le mélange $A$, Choisit $A$ (échec). il y a 1 verre d'eau de plus que de jus et dans le mélange $B$, il y a 2 verres d'eau de plus que de jus".

Voici la stratégie que met en jeu le sujet :

$$
\begin{aligned}
\text { soit } & (1,2) \text { vs. }(2,4) \\
& (1,2)=(1,1)+(0,1) \\
& (2,4)=2(1,1)+(0,2) \\
\text { or } & (1,1) \simeq 2(1,1) \\
\text { et } & (0,1)>(0,2) \\
\text { donc } & (1,1)+(0,1)>2(1,1)+(0,2) \\
\text { conclusion } & (1,2)>(2,4)
\end{aligned}
$$

La notation par couple permet de traduire le raisonnement inadéquat qu'effectue le sujet. L'erreur provient du fait que l'équivalence $\simeq$ est traitée comme une égalité $=$, et que le reste " addi. tif " n'est pas ramené au rapport de nature divisive.

Phase $b$. - Stade IIB : Schème de covariation $(a, b)$ ou de division $a / b=c / d$. Réussite des items de type $(2,4)$ vs. $(1,2)$ ou $(6,3)$ vs. $(4,2)$. 
Exemples :

Mode E (covariation des deux termes d'un rapport). Andrée, $11 ; 0$ A12: $(1,2)$ vs. $(2,4)$ "Parce qu'on a doublé Choisit $=$ (réussite). également la propor. tion d'eau et de jus".

Mode I (transposition du rapport lui-même) André, 8;0

$$
\begin{aligned}
\text { B14: }(4,2) \text { vs. }(6,3) & \text { "En } A, 4 \text { verres de jus } \\
\text { Choisit = (réussite). } & \text { pour } 2 \text { verres d'eau, } \\
& \text { c'est égal à B où il y a } \\
& 6 \text { verres de jus pour } 3 \\
& \text { verres d'eau". }
\end{aligned}
$$

Le schème, dans le mode $\mathrm{E}$, se caractérise par la covariation d'un couple à termes inégaux. Dans le mode I, il y a transposition d'un rapport. Il y a autonomie de la grandeur arithmétique des deux termes du couple, mais maintien du connecteur de conjonction dans la variation. La stratégie caractéristique du stade est la suivante:

Mode E : $m(a, b)=(m a, m b)$

$$
(a, b) \simeq(m a, m b)
$$

Mode I : $a / b=c / d$

En présence de deux rapports non équivalents à comparer, le sujet applique sa stratégie de covariation $(a, b)$ ou de transposition de rapport, et continue à isoler les restes excédentaires.

Exemple 1:

Christiane, 12;3 A23: (5,2) vs. (7,3) "Pour les deux, il y a Choisit = (échec). $\quad{ }^{1 / 2}$ verre d'eau de moins que la moitié de jus ".

Sa stratégie de résolution est la suivante:

$$
\begin{aligned}
(5,2) & =\left(5,2^{1 / 2}\right)-(0,1 / 2) \\
(7,3) & =(7,31 / 2)-(0,1 / 2) \\
\text { or }(5,21 / 2) \simeq(7,31 / 2) & \simeq \\
\text { et }(0,1 / 2) & =(0,1 / 2) \\
\text { donc }(5,2) & =(7,3)
\end{aligned}
$$


On voit que l'erreur provient de la confusion entre rapport $(2,1)$, de nature divisive, que le sujet introduit dans le problème, et " reste ", de nature additive, libéré par le traitement, dont le sujet se sert pour porter un jugement.

Exemple 2 :

Louise, 9;0

A18 : (2,1) vs. $(3,2)$ "Parce que si j'ajouChoisit $=$ (échec). tais un verre d'eau et de jus, cela donnerait la même réponse que $B$ ".

La stratégie de résolution est la suivante :

$$
\begin{aligned}
(2,1) & =(2,1) \\
(3,2) & =(2,1)+(1,1) \\
\text { or }(1,1) & \simeq(0,0) \\
\text { donc }(2,1) & =(2,1)+(1,1) \\
\text { et }(2,1) & =(3,2)
\end{aligned}
$$

L'erreur ici aussi provient d'une confusion entre rapport "divisif " et reste "additif ". L'erreur des enfants de ce stade est d'appliquer le même invariant aux deux couples en question. L'enfant ne peut encore combiner la transformation équivalente $(p \leftrightarrows q)$, permettant de construire la classe d'équivalence, avec la disjonction ( $r$ W s), permettant de comparer des couples où seul l'un des termes est multiple de l'autre, par exemple $(2,1)$ vs. $(3,2)$ sans qu'il en soit ainsi pour l'autre terme.

Phase $c$. - Stade IIIA: Schème de covariation ou équivalence logique ( $p \rightleftarrows q$ ), articulé en schème de concordance-différence ou disjonction exclusive ( $r$ W s). Réussite des items de type $(1,3)$ vs. $(2,5),(2,1)$ vs. $(3,2)$ ou $(2,3)$ vs. $(1,2)$ où deux termes correspondants des deux couples sont multiples l'un de l'autre.

Exemples :

Mode E

Danielle, 16;0 A17: (1,3) vs. (2,5) «Pour 1 verre de jus il Choisit $B$ (réussite). y a 3 verres. Pour 2 
verres de jus, il devrait $y$ avoir 6 verres. Comme il y en a que 5, le droit a un goût plus fort ".

Michèle, 12;0

A14: (2,3) vs. (1,2) "Plus d'eau que d'oChoisit $A$ (réussite). range, mais si cela avait été le double de l'eau, il aurait été supposé avoir un verre d'eau de plus (en $A)$ ".

\section{Mode I}

Nicole, $14 ; 0$ A17: (1,3) vs. $(2,5)$ " En $A$, on a 1 verre de Choisit $B$ (réussite). jus pour 3 verres d'eau, et en $B$, on a 1 verre de jus pour $2^{1 / 2}$ verres d'eau".

Diane, $14 ; 0$

A14: (2,3) vs. $(1,2)$ "Parce que dans $A$, il Choisit $A$ (réussite). y a $1 \frac{1 / 2}{2}$ verre d'eau pour 1 verre de jus, tandis que dans $B$ il y a 2 verres d'eau pour 1 verre de jus".

Dans les problèmes de ce stade, un terme d'un couple est "multiple " du terme correspondant de l'autre couple, sans que ce soit le cas pour l'autre terme. Exemple: dans $(1,3)$ vs. $(2,5)$, les premiers termes sont multiples l'un de l'autre, mais non les deuxièmes. Dans $(2,1)$ vs. $(3,2)$, les deuxièmes termes sont multiples l'un de l'autre, mais non les premiers.

Les cas où aucun des termes d'un couple n'est multiple du terme correspondant de l'autre couple sont impossibles à résoudre à ce stade, par exemple : $(3,5)$ vs. $(5,8)$. Examinons dans le détail la mobilité entre connecteurs logiques nécessaires pour résoudre les items de ce stade.

Exemples :

Danielle, 16;0 A17: (1,3) vs. (2,5) «Pour 1 verre de jus il Choisit $B$ (réussite). y a 3 verres d'eau. 
Pour 2 verres de jus, il devrait $y$ avoir 6 verres. Comme il y en a que 5, le droit a un goût plus fort".

Soit le problème $(1,3)$ vs. $(2,5)$. Première opération : covariation multiplicative $(p \rightleftarrows q)$, soit :

$$
\begin{aligned}
& p: m \cdot a=m a=c \\
& q: m \cdot b=m b=d
\end{aligned}
$$

Dans le cas particulier : $2 \cdot(1,3)=(2,6)$, l'enfant covarie les deux termes du premier couple et trouve un "équivalent " de ce couple qui lui sert comme "hypothèse " et qu'il conserve en tête pour le comparer avec le deuxième couple qui existe dans le réel.

Deuxième opération: comparaison additive $(r W$ s). L'enfant compare additivement $(2,6)$ à $(2,5)$ par concordancedifférence. Recherchons le statut logique du mécanisme de concordance-différence.

Soit deux couples $(e, f)$ et $(g, h)$

Appelons $r$ la transformation additive : $e+q=g$.

Appelons $s$ la transformation additive : $f+q=b$, où $q$ est un nombre naturel.

Nions ces transformations :

$$
\begin{aligned}
& \bar{r}: e=g \\
& \bar{s}: f=b
\end{aligned}
$$

La comparaison des deux couples $(e, f)$ vs. $(g, b)$ est possible lorsqu'il y a concordance d'un terme, différence de l'autre, ce qui correspond à une liaison logique de disjonction exclusive $(r \mathrm{~W} s)$. Il y a possibilité en effet d'établir un ordre strict entre deux couples $(e, f)$ et $(g, b)$ lorsque

mais non pas lorsque

$$
\begin{aligned}
& e=g, f+q=b, \text { soit } \bar{r} \cdot s \\
& e+q=g, f=b . \text { soit } r \cdot \bar{s} \\
& e=g, f=b, \text { soit } \bar{r} \cdot \bar{s} \\
& e+q=g, f+q=b, \text { soit } r s
\end{aligned}
$$


La table de vérité est la suivante:

\begin{tabular}{cc|c}
\hline$r$ & $s$ & $r \mathrm{~W} s$ \\
\hline 1 & 1 & 0 \\
1 & 0 & 1 \\
0 & 1 & 1 \\
0 & 0 & 0 \\
\hline
\end{tabular}

La combinaison de l'opération binaire multiplicative $(p \leftrightarrows q)$ et de l'opération binaire additive $(r \mathrm{~W} s)$ permet d'aboutir à la conclusion :

$$
(2,5)>(1,3)
$$

Il y a réussite du problème.

Phase $d$. - Stade IIIB: Algorithmes du dénominateur commun et du pourcentage. Réussite des items de type $(5,8)$ vs. $(3,5)$ ou $(7,3)$ vs. $(5,2)$ et, de façon générale, de tous les items de rapport, avec l'algorithme du dénominateur commun (mode E) ou du pourcentage (mode I).

Exemples :

Mode E

Sylvie, $14 ; 0$

A19: (2,3) vs. $(3,4)$ « $A$ droite, il y a $3 / 7$ Choisit $B$ (réussite). de jus pour $4 / 7$ d'eau, autrement dit $15 / 35$ de jus ; à gauche il n'y a que $14 / 35$ ».

Mode I

Réjean, 13;0 A23: $(5,2)$ vs. $(7,3) \lll A=713 / 7 \%$ car Choisit $A$ (réussite). $5 / 7$ du jus d'orange. $B=70 \%$ car $7 / 10$ est du jus d'orange".

Le sujet différencie d'une part la liaison logique binaire entre termes liés $(p \leftrightarrows q)$, et $(r$ W s), d'autre part les opérations arithmétiques d'addition et de multiplication élémentaire qu'il applique aux termes dissociés, pour constituer une combinatoire, ce qui lui permet de construire l'algorithme du dénominateur commun ou du pourcentage.

La stratégie de résolution d'un problème général comme 
$(a, b)$ vs. $(c, d)$ comporte les étapes suivantes (dans le mode E) :

$$
\text { Soit }(a, b) \text { vs. }(c, d)
$$

1) Addition élémentaire $(a+b)$. Addition des termes complémentaires d'un couple $(a, b)$, qui constitue effectivement un ratio, pour constituer le dénominateur d'une fraction, auquel on ramènera le premier terme du couple comme numérateur.

Soit $(a, b)$ vs. $(c, d)$

$$
\begin{aligned}
a+b=u & ; \text { addition des termes d'un même } \\
& \text { couple pour trouver le dénomi- } \\
& \text { nateur, }
\end{aligned}
$$

$$
\begin{aligned}
& \begin{aligned}
(a, b) \rightarrow & (a, u) ; \text { rapport terme à terme ou ratio ramené } \\
& \text { à rapport de numérateur à dénominateur, }
\end{aligned} \\
& \begin{array}{c}
c+d=v ; \\
(c, d) \rightarrow(c, v) ; \text { idem pour l'autre couple. }
\end{array}
\end{aligned}
$$

2) Multiplication élémentaire (u·v). Multiplication des deux dénominateurs, séparés de leurs numérateurs.

$$
\begin{gathered}
u \cdot v=v \cdot u \text { disjonction des dénominateurs, avec multiplication } \\
\text { permettant de trouver le dénominateur commun. } \\
\text { Égalité des deux produits par commutativité de la } \\
\text { multiplication. }
\end{gathered}
$$

3) Multiplication binaire ( $p \leftrightarrows q)$. Complexification de chacune des fractions pour les réduire au dénominateur commun.

$$
\begin{aligned}
& v(a, u)=(v a, v u) \\
& u(c, v)=(u c, u v)
\end{aligned}
$$

4) Addition binaire $(r W s)$. Comparaison des deux couples par concordance-différence. Les deux termes de chaque couple interviennent dans la comparaison. Il s'agit donc d'une opération binaire, avec variation disjonctive.

Soit : $(v a, v u)$ vs. $(u c, u v)$

$$
v u=u v, v a \neq u c
$$

donc $(v a, v u) \neq(u c, u v)$

Il y a ainsi formation d'une combinatoire, où les opérations logiques de conjonction et de disjonction sont applicables autant à l'addition qu'à la multiplication. 


\section{TABLEAU 6}

Les quatre phases de l'élaboration des opérations sur les rapports, à partir du schème de variation binaire: $[(1,1) \simeq(m, m)]$, durant la période d'équilibration II

(opératoire concrète et formelle)

\begin{tabular}{|c|c|c|c|c|}
\hline $\begin{array}{l}\text { Stades opéra- } \\
\text { toires piagé- } \\
\text { tiens }\end{array}$ & $\begin{array}{l}\text { Items caractéristiques } \\
(a, b) \text { vs. }(c, d)\end{array}$ & Stratégies de résolution & Mécanismes d'équilibration & $\begin{array}{l}\text { Caractéristiques des phases successives d'é- } \\
\text { quilibration }\end{array}$ \\
\hline $\begin{array}{l}\quad \text { IIA } \\
\text { Opératoire } \\
\text { concret } \\
\text { inférieur }\end{array}$ & $(1,1)$ vs. $(2,2)$ & $m(1,1)=(m, m)$ & $\begin{array}{l}\text { Centration sur les termes excéden. } \\
\text { taires, considérés indépendamment } \\
\text { de la covariation des termes du } \\
\text { couple. }\end{array}$ & $\begin{array}{l}\text { a } \\
\text { Schème de variation de termes égaux traités } \\
\text { conjointement, excluant les éléments } \\
\text { excédentaires (conjonction dans la varia- } \\
\text { tion). }\end{array}$ \\
\hline $\begin{array}{l}\quad \text { IIB } \\
\text { Opératoire } \\
\text { concret } \\
\text { supérieur }\end{array}$ & $(2,3)$ vs. $(4,6)$ & $m(a, b)=(m a, m b)$ & $\begin{array}{l}\text { Différenciation objectale, par in. } \\
\text { version de la liaison entre termes, } \\
\text { (inversion de l'égalité entre termes } \\
\text { en autonomie des termes). }\end{array}$ & $\begin{array}{l}\text { Schème de variation de termes inégaux, } \\
\text { traités conjointement. }\end{array}$ \\
\hline $\begin{array}{l}\quad \text { IIIA } \\
\text { Opératoire } \\
\text { formel } \\
\text { inférieur }\end{array}$ & $(3,1)$ vs. $(5,2)$ & $\begin{array}{l}\quad m b=d \\
m(a, b)=(m a, m b) \\
=(m a, d) \\
\quad m a>c, m b=d \\
\text { donc }(m a, m b)>(c, d) \\
\text { d'où }(a, b)>(c, d)\end{array}$ & $\begin{array}{l}\text { Différenciation opérative, par in. } \\
\text { version de la conjonction des termes } \\
\text { dans la classe d'équivalence, en va- } \\
\text { riation incompatible lors de la com- } \\
\text { paraison: ( } \\
\longrightarrow \text { ) en (W). }\end{array}$ & $\begin{array}{l}\text { Schème de covariation (ou conjonction) se } \\
\text { renverse en incompatibilité de variation, } \\
\text { lors de la comparaison entre termes des } \\
\text { deux couples après transformation. }\end{array}$ \\
\hline
\end{tabular}


La deuxième période d'équilibration est résumée au Tableau 6 , où l'on a décrit les mécanismes de passage d'un stade à l'autre pour chacune de ses phases. Précisons qu'il ne faut pas confondre les phases d'équilibration avec les périodes de développement opératoire, qui sont désignées selon la terminologie genevoise classique.

\section{INTERPRÉTATION ET DISCUSSION DES PROCESSUS EN JEU DANS LA CONSTRUCTION D'UNE NOTION}

\section{LA DUALITÉ FONCTIONNELLE ADAPTATION-ORGANISATION.}

Piaget distingue deux fonctions invariantes lorsqu'un organisme agit dans son milieu : l'adaptation de l'organisme à son milieu et l'organisation des parties dans un tout. Nous allons les examiner tour à tour.

\section{a) L'adaptation.}

L'adaptation correspond à l'interaction entre un schème et un objet adéquat. On peut la décrire par l'équilibre entre deux méca. nismes :

i) l'assimilation d'un objet familier par l'organisme, qui reconnaît en lui un aliment adéquat pour son schème et le transforme ;

ii) l'accommodation du schème au milieu spatio-temporel qui l'environne, a fin de localiser l'objet et de s'ajuster à ses dimensions.

Piaget n'a pas explicité le mécanisme qui intervient lorsque l'objet qui se présente n'est pas adéquat au schème. Les données quotidiennes conduisent à expliciter aussi les aspects négatifs de l'interaction. C'est ainsi que nous définirons l'aspect négatif de l'adaptation, lorsqu'un organisme est confronté avec un milieu inadéquat, par un équilibre entre deux mécanismes complémentaires :

iii) le rejet par l'organisme d'un objet inconnu ou superflu trouvé dans le milieu; 
iv) le retrait du schème d'un milieu défavorable, n'offrant pas d'objet adéquat au schème.

Dans un échange positif à l'équilibre, il y a toujours à la fois assimilation de l'objet par le schème, et accommodation du schème au milieu où se situe l'objet. Dans un échange négatif à l'équilibre, il y a à la fois rejet de l'objet hors de portée du schème, et retrait du schème hors du champ qui l'entoure.

Ainsi l'assimilation par le schème est solidaire de l'accommodation à l'objet, le rejet de l'objet d'un retrait du schème.

Dans un processus de recherche d'objet, lorsque se manifeste un "besoin " (ou déséquilibre intérieur), le schème, en contact avec un objet inadéquat, le rejette, sans pourtant se retirer du champ. Le déséquilibre persiste jusqu'à la découverte d'un objet adéquat, qui aboutit à la " satisfaction ", ou bien jusqu'au retrait du schème hors du champ, ce qui constitue un " renoncement ". Il y a ainsi restauration de l'équilibre.

\section{b) L'organisation}

Mais autant le schème que l'objet sont des "totalités" composées de "parties". Piaget (1936) a défini formellement l'organisation comme "rapports entre les parties et le tout ". Cependant il n'en a pas formellement explicité les mécanismes. Nous tenterons de les décrire à partir de mécanismes utilisés par Piaget.

Nous sommes d'abord conduits à définir deux mécanismes qui permettent de passer de la partie au tout et réciproquement :

i) l'intégration est l'assimilation des parties, malgré leurs différences, dans le passage des parties au tout;

ii) la différenciation est la distinction des parties, malgré leur ressemblance, dans le passage du tout aux parties.

Cependant deux nouveaux mécanismes sont nécessaires pour isoler les niveaux de la partie et du tout, et les parties entre elles:

iii) la décentration permet d'isoler le niveau du tout de celui des parties ;

iv) la séparation permet d'isoler une partie de sa complé. mentaire.

Une totalité globale ou indifférenciée en effet, ne comprend 
pas de " parties " différentes. Un double mécanisme d'isolation est nécessaire pour dissocier les parties entre elles et du tout : il s'agit de la séparation et de la décentration. Mais des parties fragmentées, à leur tour, ne possèdent pas d'unité. Un double mécanisme d'englobement est nécessaire pour les réunir, il s'agit de la différenciation entre niveau du tout et niveau des parties, et de l'intégration des parties dans le tout. Ces mécanismes fonctionnent autant au niveau du schème en relation avec ses sous-schèmes, que de l'objet en relation avec ses parties.

Ces divers mécanismes sont résumés au Tableau 7 .

\section{RELATION ENTRE LE FONCTIONNEMENT DU «SCHÈME " EN ÉQUILIBRE ET LES PROPRIÉTÉS DU “CONCEPT ».}

Afin de mieux situer les notions d'assimilation et d'accommodation dans le fonctionnement du schème : "concept en action ", on peut tenter un rapprochement avec la dichotomie compréhension-extension au niveau d'un concept élaboré.

En logique un concept est défini en comprébension par l'ensemble des caractères qui appartiennent à ce concept et qui en constituent la définition, et en extension par l'ensemble des objets auxquels il s'applique. De même en mathématique l'ensemble est défini en comprébension par une règle permettant de déterminer si un objet est membre de l'ensemble, et en extension par une liste des éléments de l'ensemble.

C'est ainsi que l'ensemble " des nombres premiers plus petits que dix " peut être représenté par une description de l'ensemble, telle que :

$$
A=\{x / x \text { est un nombre premier à un chiffre }\}
$$

ou par le procédé de l'énumération :

$$
A=\{2,3,5,7\}
$$

Lorsqu'un sujet applique un concept à des données du milieu, nous allons considérer le sujet, qui agit au moyen d'un schème, comme siège de la compréhension du schème, le milieu, comprenant des objets discrets ou en étendue, comme support de l'extension du schème. 


\section{TABLEAU 7}

Les mécanismes propres des deux fonctions de l'organisme: adaptation et organisation

\begin{tabular}{|l|c|l|l|}
\hline \multirow{2}{*}{ Fonctions } & \multirow{2}{*}{$\begin{array}{c}\text { Direc- } \\
\text { tion }\end{array}$} & \multicolumn{1}{|c|}{ Pôle d'assimilation } & Pôle d'accommodation \\
\cline { 3 - 4 } & + & $\begin{array}{l}\text { Assimilation généralisa- } \\
\text { trice de l'objet connu. }\end{array}$ & $\begin{array}{l}\text { Accommodation particulari- } \\
\text { sante à l'objet actuel. }\end{array}$ \\
\hline \multirow{3}{*}{ Adaptation } & - & Rejet de l'objet étranger. & Retrait de l'objet étranger. \\
\cline { 2 - 4 } & + & $\begin{array}{l}\text { Assimilation intégrative } \\
\text { des parties. }\end{array}$ & $\begin{array}{l}\text { Accommodation différencia- } \\
\text { trice aux parties. }\end{array}$ \\
\hline \multirow{2}{*}{ Organisation } & - & Décentration d'une partie. & Séparation des parties. \\
\cline { 2 - 4 } & &
\end{tabular}

Ainsi l'on pourra transcrire un schème $S$ par la description en compréhension de l'ensemble $A$ qu'il engendre (où $=>$ signifie " engendre ")

$$
S=>A
$$

$A=\{x / x$ est un nombre premier à un chiffre $\}$

Le schème sera ainsi défini en compréhension par son "assimilation", ou l'ensemble qu'il détermine dans le milieu. Mais on pourra aussi décrire le schème en extension, par les objets auxquels il peut s'appliquer, soit son " champ d'accommodation ":

$$
\begin{aligned}
& S=>A \\
& \mathrm{~A}=\{2,3,5,7\}
\end{aligned}
$$

Comme le sujet agit bic et nunc, sur un objet à la fois, cela nous amène à distinguer :

i) l'extension actuelle d'un schème, ou son accommodation locale

ii) l'extension totale d'un schème, ou son champ d'accommodation.

En effet l'interaction entre un schème et les objets actuels du milieu est une activité localisée, et non nécessairement exhaustive. Le champ d'accommodation, au contraire, correspond à 
l'extension totale du schème. Prenons un exemple. Devant le schème décrit plus haut :

$S=>A$

$\mathrm{A}=\{x / x$ est un nombre premier à un chiffre $\}$

le sujet, placé devant l'objet 2 , reconnaîtra cet objet comme, adéquat au schème (c'est l'assimilation généralisatrice de Piaget), et l'incorporera ainsi dans l'ensemble concerné. Placé devant l'objet 5 , le sujet fera de même. Les objets 2 et 5 , qui étaient de simples " nombres naturels ", ont acquis une signification nouvelle en étant incorporés par le schème, ils sont devenus chacun "un nombre premier à un chiffre". Le mécanisme de cette transformation est l'“assimilation". Devant l'objet 6, au contraire, le sujet le rejettera hors de l'ensemble. Nous parlerons ici de rejet de l'objet 6 , introduisant le mécanisme de rejet, inverse de l'assimilation.

On constatera que dans chaque cas particulier, le schème générique d'assimilation, défini en compréhension comme indiqué ci-dessus, se "particularisera" pour appréhender l'objet particulier. En effet, dans l'expression :

$$
A=\{x / x \text { est un nombre premier à un chiffre }\}
$$

$x$ est une "variable ". Le symbole $x$ représente tous les éléments de l'ensemble à définir et susceptibles d'être appréhendés par le schème. Cependant chacun de ces éléments implique une valeur définie de la variable. Le processus de " particularisation ", chaque fois qu'il y a récognition d'un élément nouveau, correspond au mécanisme d'" accommodation" de Piaget. Nous parlerons d'accommodation particularisante. Le schème générique assume par accommodation particularisante une valeur individuelle correspondant à la situation présente. S'il y avait correspondance terme à terme entre activité de l'organisme et objets du milieu, le réglage d'assimilation-accommodation, avec son complémentaire de rejet-retrait, ne serait pas nécessaire. Mais comme le sujet se trouve placé devant un milieu dont il est devenu "autonome", dont il s'est " libéré ", la recherche des objets adéquats à sa survie ou son activité intellectuelle, nécessite un réglage, au moyen de mécanismes exploratoires, que Piaget a décrit sous le nom d'assimilation-accommodation. Ces fonctions marquent ainsi la "liberté " relative de l'organisme, mais aussi sa " solidarité ", face 
au milieu. Le mécanisme d'assimilation généralisatrice sera l'incorporation d'un objet par le schème, ce que l'on pourra traduire, pour le schème décrit, par :

\section{$2 \in A$}

Le mécanisme d'accommodation particularisante consistera dans la particularisation du schème en fonction d'un objet donné, ce qui sera représenté comme suit :

\section{$A \ni 2$}

où l'on introduit un signe $\ni$ qui signifie "comprend", qui est l'inverse du signe " appartient à ".

En logique et en mathématique on décrit toujours des situations d'équilibre, où la compréhension d'un concept correspond à son extension. Cette situation existe chez l'enfant lorsqu'il possède un schème qui s'applique exhaustivement aux objets qu'il manipule. Le sujet est en équilibre avec le milieu.

L'équilibre entre sujet et milieu, dans une situation donnée, se traduit par une adéquation entre la compréhension du schème et son extension dans la situation envisagée (par une adéquation entre assimilation et accommodation). Le mécanisme mis en jeu pour qu'un schème d'assimilation s'accommode à chaque situation particulière est la forme d'équilibration que nous pouvons appeler équilibration simple ou équilibration intrastade.

\section{DÉSÉQUILIBRE APPORTÉ PAR LE MILIEU LORSQUE L'OBJET DEVIENT " COMPOSÉ "}

Mais il survient des situations où le sujet se heurte à un objet " composé ", qui résiste partiellement à l'appréhension par le sujet, parce que la structure de cet objet déborde la compréhension du schème. Tel est le cas, par exemple, où un sujet, qui possède la notion de nombre naturel, se heurte à un nombre rationnel. Le sujet pourra appréhender ce nouvel objet en tant que nombre, le reconnaitre comme familier sous certains aspects, mais d'autres aspects lui échappent. Le sujet ne maîtrise plus totalement la réalité qu'il a devant lui. Il y a déséquilibre entre sujet et milieu.

Le processus que Piaget appelle: équilibration majorante correspond aux mécanismes permettant au sujet de passer d'un 
objet simple, saisi exhaustivement au moyen d'un schème connu, à un objet "composé ", dont une partie échappe au schème et crée une "perturbation ». Quelles en sont les modalités ?

Le paradigme qui se dégage des expériences effectuées est une interaction constante entre sujet et milieu, avec restructuration récriproque. Le milieu est support de l'objet composé dont les propriétés sont autonomes, et dont les relations entre parties, pour constituer le tout, dépendent de conditions physiques du milieu. Mais l'organisme aux prises avec les objets nouveaux du milieu agit à partir de ses propres schèmes d'activité, et reconstruit son schème à partir de son action sur ce milieu. Cependant, c'est le milieu qui présente les résistances et se trouve ainsi à diriger l'activité de l'organisme et ses compositions nouvelles dans des directions déterminées. Aussi l'adaptation entre sujet et milieu n'est possible que lorsque le milieu produit des compositions entre parties qui sont compatibles, donnant ainsi naissance à des sousensembles complémentaires à l'intérieur d'un ensemble, ce que reproduit l'adaptation par son mécanisme d'inversion du schème pour donner un schème bidirectionnel ou réversible. Le schème d'assimilation de l'organisme s'adaptera à son environnement nouveau dans la limite où il pourra reproduire en lui-même, (sous forme d'abstraction réfléchissante), la combinaison entre parties que le milieu effectue. La compatibilité entre parties à l'intérieur d'un tout, existant dans le milieu, entraînera la composition de sous-schèmes à l'intérieur du schème au niveau de l'organisme. L'organisme demeure siège de l'activité de reconstruction, mais cette reconstruction est constamment dirigée par les repères que lui présente le milieu. C'est ainsi que nous adoptons une interprétation peut-être plus " réaliste " que celle de Piaget, en ce qui concerne l'origine des "perturbations".

Pour qu'un processus d'équilibration s'amorce à partir du sujet, l'aspect nouveau de l'objet, prétendons-nous, doit être composable avec l'aspect ancien, à l'intérieur même du réel. Cette composition, une fois "comprise" par le sujet, devient une " complémentarité " de parties, mais, avant son " assimilation", elle est aperçue comme une "contradiction", interprétation dialectique de ce que la philosophie classique appelait " composition". Si le sujet n'appréhende dans le réel qu' une partie donnée de l'objet, la contrepartie constitue une "contradiction" 
actuelle, et cela tant que le sujet n'aura pas intégré partie et contrepartie, dans une totalité hiérarchisée et composée. Cela s'effectuera par le processus d'équilibration majorante.

\section{L'ÉQUILIBRATION MAJORANTE ET LA RÉORGANISATION DU SCHEME EN FONCTION DU MILIEU}

\section{a) L'équilibration majorante et la construction d'une biérarcbie.}

Le passage d'un stade à l'autre, dans le développement d'un schème, est la modification dans la compréhension du schème afin d'appréhender l'extension nouvelle du donné. Nous interpréterons la différenciation comme la modification de la compréhension du schème en fonction du donné. Nous interpréterons de même l'intégration comme l'incorporation en un tout des deux directions inverses du schème, permettant d'appréhender les éléments nouveaux du donné, à côté des éléments anciens, pour saisir l'objet total présenté par le milieu comme un tout hiérarchisé, où la totalité se différencie des parties, mais les englobe. Ce problème de passage d'un stade au suivant étant relativement nouveau, nous allons l'examiner un peu en détail.

Le schème est en effet une activité cognitive, un " concept en action ", qui appréhende les éléments du milieu qui lui sont adéquats (formant ainsi un ensemble), et se retire des éléments inadéquats. Si l'on élargit l'ensemble par des éléments étrangers qui en augmentent l'extension, il s'agira pour le sujet de modifier la compréhension de son schème, s'il veut saisir ces éléments nouveaux. Il est amené ainsi à construire une biérarchie.

Une hiérarchie est à la fois ensemble et ordre, et cela autant pour le schème que pour son substrat. Au niveau du schème, quant à l'aspect ensemble, la hiérarchie est constituée d'un schème générique, qui incorpore les deux directions opposées des sousschèmes spécifiques (appréhender-rejeter). Quant à l'aspect ordre du schème, il $\mathrm{y}$ a distinction entre schème et sous-schème, et inversion de direction dans le passage d'un sous-schème à l'autre. Au niveau du substrat, la hiérarchie est constituée d'un ensemble englobant à un niveau générique et de sous-ensembles complémentaires à un niveau spécifique. Elle présente une distinction d'ordre entre les niveaux de l'ensemble et des sous- 
ensembles et une complémentarité dans le passage d'un sousensemble à l'autre.

Aussi la " construction " d'une hiérarchie s'effectue-t-elle par l'intervention de plusieurs mécanismes, correspondant aux différentes composantes de l'interaction entre schème et donné. On peut les ramener à deux séries, qui agissent solidairement, et permettent de passer du simple au hiérarchisé. Les deux mécanismes d'ordre sont la décentration du schème, par rapport au donné, et l'inversion du schème, dans le passage de la partie connue à la partie nouvelle du donné. Les deux mécanismes d'ensemble sont la différenciation du schème en deux sousschèmes, et l'intégration des deux sous-schèmes en un schème nouveau.

Le substrat résultant de cette restructuration reflétera les distinctions établies au niveau du schème.

b) Les étapes de l'équilibration majorante.

Le schème à sens unique fonctionne à un seul niveau : il y a assimilation de l'objet connu, ou son inverse : rejet de l'objet superflu ou inconnu. Le schème, aux prises avec un objet composé, se trouve d'abord dans un état de déséquilibre, une partie du donné étant appréhendée, l'autre omise. Un premier mécanisme de restructuration est un mécanisme d'ordre, qui permet au sujet de prendre ses distances par rapport au donné : de dissocier partie centrée et totalité du donné. C'est la décentration, qui introduit une distinction d'ordre entre tout et parties, et amène le sujet à modifier son schème dans son extension. Le schème, qui ne s'appliquait qu'à la partie connue du donné, doit alors se différencier quant à ses critères, pour appréhender à la fois la partie nouvelle et la partie ancienne, à l'intérieur d'une totalité qui comprend tout le donné.

Parallèlement, un second mécanisme d'ordre : l'inversion, permet au sujet de passer de la partie assimilée à la partie omise du donné, en appliquant son schème dans les deux directions.

Un mécanisme d'ensemble: l'intégration, permet au sujet d'incorporer le mouvement direct d'assimilation, de la partie connue du donné, et inverse de rejet, de la partie inconnue, en une totalité composée correspondant au schème bidirectionnel : à 
la fois direct et inverse. En effet, le schème, aux prises avec un objet composé, dont les parties ont des effets inverses sur la totalité qu'elles composent, ne peut s' "accommoder " à chacune de ces parties, que s'il inverse son mouvement selon la partie en jeu. Comme exemple, prenons le schème d'évaluation du goût en orange d'un mélange : le sujet ayant intégré les deux mouvements inverses considérera que la valeur du goût en orange augmente en fonction de la quantité de jus, et diminue avec la quantité d'eau. En terminologie piagétienne, le schème est devenu "réversible " (bidirectionnel), et la représentation "décentrée " (hiérarchisée). Selon la terminologie de Heinz Werner, il y a «intégration hiérarchique".

Décentration, inversion, différenciation et intégration sont des aspects solidaires d'un même processus d'équilibration. La décentration, en libérant le pôle du sujet de ses liens étroits avec l'objet, permet de considérer la composante négative de l'objet composé, comme égale "en signification", mais différente "en direction de variation ", de la composante positive. Le" schème pourra ainsi "assimiler" la composante négative à l'égal de la composante positive, à l'intérieur d'un objet total. Cela constitue le mécanisme d'assimilation intégrative, solidaire de la différenciation antagoniste, permettant la construction d'une hiérarchie et passage d'un stade au suivant.

\section{c) Illustration par l'expérience décrite.}

Prenons comme exemple l'expérience décrite.

Soit $J$ un ensemble de verres de jus d'orange, dont la quantité est indéterminée,

$$
J=\{x / x \text { est un nombre naturel }\}
$$

L'on définit l'ensemble $E$ de verres d'eau,

$$
E=\{y / y \text { est un nombre naturel }\}
$$

L'on définira l'ensemble produit $J X E$,

$$
J X E=\{x / y / x \in J \text { et } y \in E\}
$$

L'ensemble produit $J X E$ correspond au " mélange " de verres de jus d'orange et d'eau en même quantité, soit aux rapports : $1: 1$, $2: 2,3: 3$, etc. Par quels mécanismes l'enfant passera-t-il pour 
construire la notion de rapport $x / y$, lorsqu'il possède la notion de $x$ et de $y$ ?

Nous voyons qu'il lui faut passer par une succession de phases, où interviendra un mécanisme d'" assimilation intégrative". Ceci nous amène à distinguer assimilation généralisatrice et assimilation intégrative.

Comme nous l'avons vu, l'assimilation généralisatrice consiste à incorporer un objet particulier dans un ensemble $A$ :

$2 \in A$

L'assimilation généralisatrice, appliquée aux objets de deux ensembles, $J$ et $E$ de verres de jus d'orange et d'eau, consistera à reconnaître dans ceux-ci des éléments de ces ensembles.

Devant une juxtaposition de deux verres de jus d'orange et de trois verres d'eau, par assimilation généralisatrice, l'enfant établira ce qui suit :

$$
2 \in J
$$

et dira "Il y a 2 verres de jus d'orange, il y a 3 verres d'eau". Lorsqu'on "mélange " jus d'orange et eau pour former un "objet composé ", les transformations suivantes sont nécessaires :

Décentration: Au lieu de considérer 2:3, par exemple, comme deux nombres naturels d'objets différents, l'enfant devra se distancer de chacun d'eux en particulier, pour considérer le tout que forme un mélange distinct des parties. Il y a constitution d'un ensemble nouveau $J X E$, dont les éléments sont des rapports $e$ correspondant à un "goût " en jus d'orange.

Différenciation antagoniste: L'ensemble nouveau $J X E$ est vu comme constitué de deux sous-ensembles complémentaires $E=J$ '.

Inversion : Si l'effet des verres de jus sur l'ensemble est vu comme augmentant le goût du jus, l'effet de verres d'eau est vu comme le diminuant. Il y a inversion de sens dans la fonction qui relie le goût en mélange et la cardinalité de chacun des sous-ensembles.

$$
x=1 / y
$$

Assimilation intégrative: Mais ces deux composantes combinent 
leur effet dans une totalité nouvelle, le "mélange ", dont chaque élément est un goût particulier ou "rapport ":

$$
\mathrm{J} \mathrm{X} \mathrm{E}=\begin{gathered}
e \in J X E \\
x / y / x \in J \text { et } y \in E \text { et } x=1 / y\} \\
e=x / y
\end{gathered}
$$

C'est ainsi que si l'assimilation généralisatrice porte sur les éléments d'un ensemble auxquels elle accommode un schème unidirectionnel pour les identifier, l'assimilation intégrative porte sur les éléments d'un ensemble produit qu'elle appréhende par un schème devenu "réversible ". L'ensemble de ces mécanismes correspond à l'équilibration majorante.

\section{d) L'aspect constructiviste de l'équilibration majorante.}

Une théorie du développement, basée sur le mécanisme de l'équilibration majorante, n'est pas réductionniste, puisqu'elle ne réduit pas le complexe au simple. Mais étant interactionniste, elle montre comment le complexe est construit, à partir du simple, grâce à une combinaison d'assimilation et d'ordre. La décentration, qui est distinction de niveaux, permet d'intégrer les inversions de mouvement à l'intérieur d'une totalité hiérarchisée. L'organisme qui incorpore l'inverse de son mouvement d'assimilation, à l'intérieur d'un schème devenu réversible, maîtrise l'objet " extrinsèque ", qu'il appréhendait auparavant par un schème étranger.

Dans l'équilibre du stade précédent, l'assimilation était en équilibre avec le rejet, il y avait assimilation de l'objet connu et rejet de l'objet superflu ou inconnu. Dans l'équilibre nouveau qui s'instaure, le sujet reproduit en lui-même, comme double "intrinsèque ", l'objet composé extérieur, en transformant son propre mouvement d'appréhension et de retrait au contact de l'objet, en un mouvement d'accommodation à la fois directe et inverse, intégré par assimilation même au niveau du schème, qui se trouve ainsi être en équilibre avec les deux composantes de l'objet.

C'est la dualité : assimilation-rejet, combinée à la distinction de niveau, qui permettra de construire, à l'intérieur du sujet, le double de l'objet qu'est la "notion " que le sujet en tire. La construction de la notion se fait "à l'intérieur " du sujet, mais "à propos " de l'objet, par action (positive) sur l'objet et rejet 
(négatif) de l'objet, enfin combinés dans un schème unique. Le " complexe " dépasse le " simple " puisqu'il n'est pas seule union de simples, mais intégration des mouvements inverses accomplis sur le simple.

5. LES DEUX MODALITÉS D'ÉQUILIBRATION : A L'INTÉRIEUR D'UN STADE ET DANS LE PASSAGE D'UN STADE AU SUIVANT.

Aux périodes d'équilibre, adaptation et organisation fonctionnent solidairement. Lors des déséquilibres nécessitant la restructuration du schème, le passage d'un stade au suivant s'effectue par une articulation nouvelle de ces deux fonctions, l'adaptation, source de déséquilibres, fournissant la direction des mouvements requis pour cerner les aspects nouveaux de la réalité, l'organisation, permettant d'incorporer ces divers mouvements dans une totalité hiérarchisée.

L'équilibration, à l'intérieur du stade, est le fonctionnement d'un schème déjà construit, qui s' " actualise " par généralisation et particularisation. L'équilibration, dans le passage d'un stade au suivant, est une réorganisation du schème, où l'on retrouve les mêmes mécanismes que dans le fonctionnement intra-stade, mais " en genèse ".

La particularisation du schème en fonction de l'objet, (appartenant au sous-ensemble principal), prédomine dans le premier mode, le rejet de l'objet, (faisant partie du sous-ensemble complémentaire), s'effectuant d'emblée. La hiérarchie est déjà construite.

L'intégration des objets du sous-ensemble complémentaire dans un ensemble total prédomine dans le deuxième mode, provoquant la "genèse " d'une hiérarchie. Ces deux variantes d'équilibration sont résumées au Tableau 7.

\section{LES QUATRE PHASES DE L'ÉQUILIBRATION MAJORANTE $A$ LA PREMIÈRE PÉRIODE.}

Comme l'interaction entre sujet et objet comporte deux niveaux, celui du schème et celui de l'objet, la réorganisation entraînée par l'apparition d'une composante nouvelle dans l'objet comportera des aspects tant objectal (au niveau de l'objet), 
qu'opératif (au niveau du schème). L'on trouve en effet que l'assimilation, par le schème, d'un aspect nouveau dans l'objet, s'effectue en quatre phases:

a) L'on a, au départ, un état initial d'équilibre entre schème et objet, caractérisé par l'assimilation par le schème de l'objet dans son aspect connu, et retrait du schème de l'aspect étranger de l'objet.

b) Il y a ensuite incorporation, par le schème, de l'aspect nouveau de l'objet, sous forme d'élément inverse ; l'aspect nouveau trouvé dans l'objet est assimilé à l'aspect ancien, à titre de composante exerçant une influence inverse sur le schème, qui s'élargit ainsi, pour traiter des composantes soit directes, soit inverses. C'est la phase de différenciation objectale.

c) Il y a ensuite mise en relation entre la variation directe de l'objet et la variation inverse. La relation se détache des termes pour devenir mobile. C'est la phase de différenciation opérative.

d) Il y a enfin différenciation entre la relation entre termes et la variation des termes. La relation entre termes est stabilisée sous forme d'invariant; la variation des termes est intégrée sous forme de covariation, pour constituer ensemble une "opération". Celleci devenue mobile porte sur des objets "composés". Il y a constitution d'une totalité opératoire.

\section{LES DEUX PÉRIODES DE L'ÉQUILIBRATION MAJORANTE.}

Un processus d'équilibration majorante à quatre phases se retrouve à chacune des périodes de développement de l'enfant. Il est illustré dans l'expérience présente par la constitution du nombre rationnel, puis des opérations sur les rationnels, à deux périodes successives de développement, préopératoire et opératoire. Ce qui était appliqué à des transformations "unaires" à la première période, est appliqué à des transformations "binaires" à la seconde.

À la première période, préopératoire, le point de départ est le schème de variation "élémentaire " d'un terme. Il y a, dans une deuxième phase, incorporation d'un deuxième terme, comme inverse du premier terme ; dans une troisième phase, il y a mise en relation de ces termes, pour constituer, à la quatrième phase, le 
nombre rationnel, ou rapport entre termes, ceux-ci variant conjointement à l'intérieur de la "classe d'équivalence ".

À la deuxième période, opératoire d'équilibration, le point de départ est le schème de variation " binaire " de deux termes égaux du rapport. La classe d'équivalence correspond à la covariation ou variation équivalente des deux termes du rapport. Dans une deuxième phase, les deux termes deviennent autonomes quant à la grandeur. $\grave{A}$ une troisième, il y a articulation de la variation binaire d'équivalence des termes, avec son inverse la disjonction exclusive. $\hat{A}$ une quatrième phase, il y a constitution de l'ensemble des opérations sur les rationnels, combinant la classe d'équivalence avec les opérations sur termes isolés.

Durant la première période d'équilibration, une variation directe se transforme en variation inverse pour se combiner avec elle en un rapport covariant de termes égaux ; durant la deuxième, un rapport de termes égaux se transforme en un rapport de termes inégaux pour donner enfin des rapports quelconques.

Nous pourrions résumer cette construction de totalités nouvelles selon quatre phases successives d'équilibration en réinterprétant les phases du mouvement dialectique :

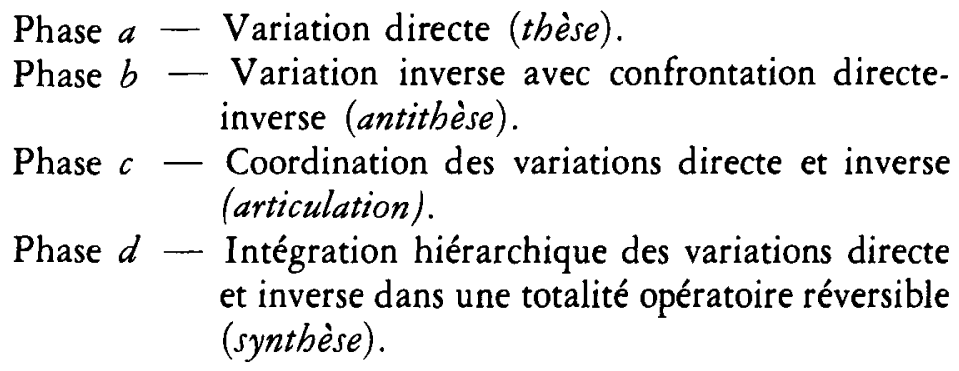

\section{CONCLUSION}

Le constructivisme piagétien se révèle ainsi, tout à la fois, "dialectique " dans la description des processus d'adaptation de l'organisme au milieu, mais " organismique " dans l'organisation des parties dans le tout, lorsque l'organisme est confronté avec des objets "composés". C'est le milieu qui présente le substrat nouveau qui déséquilibre l'organisme et le provoque à s'adapter, mais la reconstruction du schème pour faire face à la situation 
nouvelle est dictée par les possibilités de l'organisme, et s'effectue selon une séquence logique où l'on reconnaît les mécanismes propres au schème. Si l'inversion du schème est provoquée par le milieu, l'incorporation de cette inversion dans une totalité nouvelle est due à l'organisme. L'articulation constante de l'adaptation et de l'organisation explique le développement des organismes aux prises avec un milieu changeant.

École de Psychologie,

Université Laval.

\section{RÉFÉRENCES}

DIXON, W.J. (1971) Ed., Biomedical Computer Programs, Berkeley, University of California Press.

GuTTMAN, L. (1947), "The Cornell technique for scale and intensity analysis", Educ. Psychol. Measmt., 7, 247.280.

NoeltiNG, G. et al., Les mécanismes d'équilibration dans la construction de la notion de proportion chez l'enfant et l'adolescent, (en préparation).

PIAGET, J. (1936), La naissance de l'intelligence cbez l'enfant, Neuchâtel, Delachaux et Niestlé, 7e édition, 1970.

PIAGET, J. (1967), Biologie et Connaissance, Paris, Gallimard.

PIAGET, J. (1968), Le structuralisme, Paris, P.U.F.

PIAGET, J. (1975), L'équilibration des structures cognitives, problème central du développement, Paris, P.U.F.

WHITE, W. \& SALTZ, E. (1957), "Measurements of reproducibility ", Psychological Bulletin, 54, 81-99. 